\title{
Pyrene appended bile acid conjugates: Synthesis and a structure-gelation property study
}

\author{
SHREEDHAR BHAT $^{\mathrm{a}, \mathrm{b}}$, ARTO VALKONEN ${ }^{\mathrm{a}}$, JUHA KOIVUKORPI ${ }^{\mathrm{a}}$, ANUPAMA AMBIKA ${ }^{\mathrm{b}}$, \\ ERKKI KOLEHMAINEN $^{\mathrm{a}, *}$, UDAY MAITRA ${ }^{\mathrm{b}, *}$ and KARI RISSANEN ${ }^{\mathrm{a}, *}$ \\ aDepartment of Chemistry, Nanoscience Center, University of Jyväskylä, 40014 Jyväskylä, Finland \\ ${ }^{\mathrm{b}}$ Department of Organic Chemistry, Indian Institute of Science, Bangalore 560 012, India \\ e-mail: erkki.t.kolehmainen@jyu.fi; maitra@orgchem.iisc.ernet.in; kari.t.rissanen@jyu.fi
}

MS received 19 January 2011; accepted 11 March 2011

\begin{abstract}
A wide variety of novel compounds obtained by combining two types of known organogelators, viz., bile acid alkyl amides and pyrene alkanoic acids, were synthesized and screened for their gelation ability. The $3 \alpha$ esters of 1-pyrene butyric acid (PBA) of alkylamides of deoxycholic acid (DCA) turned out to be effective in the gel formation with many organic solvents although the gelation has to be triggered by the addition of a charge transfer (CT) agent 2,4,7-trinitrofluorenone (TNF). The special feature of these molecules is that the organogelation is achieved only after derivatizing the acid moiety of the 1-pyrenealkanoic acids. Additionally, the gelation properties can be fine-tuned by inserting different functional groups at the bile acid side chain. The gels obtained are deep red in colour and optically transparent up to $2 \% \mathrm{w} / \mathrm{v}$. The SEM studies of the obtained xerogels revealed bundled rod-like morphology without specialized branching.
\end{abstract}

Keywords. Pyrene; bile acid; 2,4,7-trinitrofluorenone; two component gel; scanning electron microscopy.

\section{Introduction}

'Gels' are classified in many ways. ${ }^{1}$ An 'organogel' is a poorly-crystalline, non-glassy thermoreversible solidlike material composed of a liquid organic phase entrapped in a structured network. ${ }^{2}$ The liquid can be e.g., an organic solvent, a mineral oil or a vegetable oil. Organogels can be either derived from a polymer or from small molecules. These molecules self-assemble into elongated fibrous structures through highly specific molecular interactions in an organic solvent. The gelling agents, so called 'small molecule gelling agents' are gaining popularity in the gelling of a wide range of organic solvents to form organogels, ${ }^{3}$ which are attractive materials for many applications. Many of the low molecular weight organogelators (LMOGs) display thixotropy - they become fluid when agitated, but resolidify when resting. During the past two decades, a wide range of LMOGs have been discovered that range from alkanes ${ }^{4}$ to alkanoic acids, steroids to vitamin $C,{ }^{5}$ nucleosides to DNA. ${ }^{6}$ The gelation process necessarily creates novel fibrous supramolecular structures which can be characterized by microscopy and scattering

${ }^{*}$ For correspondence studies. Applications of organogels include, among many others, ${ }^{7}$ drug delivery. ${ }^{8}$ By replacing the liquid with a gas it is possible to prepare aerogels, materials with exceptional properties including very low density, high specific surface areas, and excellent thermal insulation properties. ${ }^{9}$

The exact mechanism of the gelation of organic solvents by LMOGs is not known. Although it is generally accepted that intermolecular forces such as $\mathrm{H}$ bonding, ${ }^{10}$ electrostatic attractions and/or $\pi-\pi$ stacking interactions are necessary to stabilize LMOG assemblies. It has also been demonstrated that London dispersion forces alone can be sufficient. ${ }^{11}$ The large variety of structurally different gelators show that H-bonding, but also electrostatic, $\pi-\pi$ or charge transfer interactions may lead to self-assembling networks build up from long often entangled fibres (whiskers). ${ }^{12}$ Furthermore, it has been shown that appropriate gelators can physically form liquid crystalline phases. ${ }^{13}$

The gelation properties can be modified by both chemical and physical means. Chemically, the gelation process can be controlled by $\mathrm{pH}$, ionic strength and additives. ${ }^{14}$ Stimuli-responsive assembly of low molecular weight materials $(\mathrm{MW}<1000)$ have been intensively studied in gels, micelles, vesicles, and synthetic membranes. ${ }^{15}$ Sound, ${ }^{16,17}$ light $^{18-20}$ and electrochemical 
controls $^{21}$ have been explored as a means of switching molecular aggregations without the use of chemical stimuli. However, providing a method of control that is instant, positive (i.e., converting sol to aggregate), and reversible, while also being versatile and practical, remains a challenge.

Moreover, organogels commonly suffer from instability, for instance, due to crystallization or lack of mechanical robustness. Furthermore, despite major achievements in supramolecular chemistry to achieve controlled self-assembly of organic molecules, ${ }^{22}$ until now most low molecular weight gelators have been found by serendipity rather than by design. Recently, Hanabusa et al. ${ }^{23}$ Feringa et $a l .{ }^{24}$ as well as Maitra et $a l .{ }^{25}$ succeeded in the systematic design of novel gelators for organic solvents by exploiting the selfassembling properties of amide, urea and urethane groups. $^{26}$

Our interest on the design of new materials prompted us to connect the existing gelator motifs into hybrid structures and study their gelation properties in solution. We envisioned that by adding a pyrene moiety into a bile acid derived organogelators would lead to an interesting new type of molecules that could give rise to novel self-assembling systems. Our previous reports introduce CT gelation of pyrene analogues. In this article, we describe the 'structure-gelation' property of a new class of gelators designed by covalently linking the two well-known gelator moieties (viz., derived from pyrene and bile acid).

\section{Experimental}

\subsection{Methods}

2.1a Spectroscopy: Absorption spectra were recorded on a Shimadzu UV2100 spectrometer equipped with a thermoelectric variable-temperature set-up. FTIR spectra were recorded on a Jasco-70 FT-IR spectrometer or on a Perkin Elmer Spectrum GX FT-IR spectrophotometer either by making a thin film of the compounds on a $\mathrm{NaCl}$ plate from a chloroform solution or using $\mathrm{KBr}$ pellets. One-dimensional ${ }^{1} \mathrm{H},{ }^{13} \mathrm{C}$, and ${ }^{13} \mathrm{C}$ DEPT-135 NMR spectra were recorded in dilute $\mathrm{CDCl}_{3}$-solutions at $30^{\circ} \mathrm{C}$ with Bruker Avance DRX 500 (or DPX 250) FT NMR spectrometer equipped with an inverse detection dual probehead (BBI) and z-gradient accessory working at $500.13 \mathrm{MHz}$ for proton and at 125.77 for carbon-13, respectively. The ${ }^{1} \mathrm{H}$ and ${ }^{13} \mathrm{C}$ chemical shifts were referenced to the signal of internal TMS $(\delta=0.00 \mathrm{ppm})$. Variable temperature (VT) H and
${ }^{13} \mathrm{C}$ NMR spectra were recorded on a JEOL $300 \mathrm{MHz}$ spectrometer. ESI-QTOF MS spectra were recorded on a Micromass Q-TOF micro mass spectrometer. The gelation tests were carried out in pyrex test tubes with a diameter of $8 \mathrm{~mm}$ and a length of $70 \mathrm{~mm}$.

2.1b Gelation tests: The compound and powdered TNF (typically 1:1, $\mathrm{C}_{\text {gelator }} 1 \% \mathrm{w} / \mathrm{v}$ ) were mixed in a test tube. The suspension in the corresponding solvent was warmed on a water bath maintained at $90^{\circ} \mathrm{C}$ until all the solid material dissolved forming a clear solution. The hot sol was allowed to gel at ambient temperature without external disturbance. For SEM imaging, the samples were prepared by placing $10 \mu \mathrm{L}$ of a hot solution on a carbon tape pasted on sample stubs, and after drying the samples for $24 \mathrm{~h}$ they were subjected to gold coating using DC sputtering $(50 \AA)$ for $38 \mathrm{~s}$ and examined using a QUANTA 200 scanning electron microscope with a single crystal of LaB6 as the emitter.

2.1c Gel melting: The gel-sol transition behaviour was characterized by gel melting. The gel melting was estimated by the 'inverted test tube' method. Briefly, gels were made in $8 \mathrm{~mm} \times 70 \mathrm{~mm}$ tubes, sealed at the open end, and placed upside down in a water/oil bath whose temperature was increased slowly $\left(2^{\circ} \mathrm{C} / \mathrm{min}\right)$. The temperature at which the gel fell was recorded as $\mathrm{T}_{\text {gel,-sol }}$ which was usually within $\pm 2^{\circ} \mathrm{C}$ in multiple measurements.

\subsection{Materials}

2.2a General: All the glassware were acid rinsed prior to wash with deionized water and dried in a hot oven. All the organic solvents were analytical grade or were distilled prior to use. Chloroform and dichloromethane were distilled over $\mathrm{CaH}_{2}$, THF and toluene over sodium/benzophenone ketyl, and pyridine and $\mathrm{Et}_{3} \mathrm{~N}$ over $\mathrm{KOH}$, respectively. All the reagents were purchased from Aldrich and used as received. 1Pyrene carboxylic acid and 1-aminopyrene were prepared starting from pyrene as reported earlier. ${ }^{23 \mathrm{~b}} 1$ Pyrene butyric acid and 1-pyrene dodecanoic acid were prepared according to our previous reports. ${ }^{27}$ TLC was performed on pre-coated silica gel plates (Merck) and stained with iodine vapour, or with LB (Liebermann Buchard) stain, or observed under UV light. Column chromatography was carried out on $100 \pm 200$ mesh silica gel on gravity columns. 
2.2b Compound 4: 3,7,12-Triformylcholic acid $\left(0.25, \quad 0.50 \mathrm{mmol}, \quad\right.$ m.p. $\left.\quad 204-205^{\circ} \mathrm{C}\right),{ }^{28}$ hexadecyalamine $(0.15 \mathrm{~g}, 0.62 \mathrm{mmol})$ and DMAP $(6 \mathrm{mg}$, $0.05 \mathrm{mmol}$ ) were dissolved in dry DCM. To this stirring mixture was added DCC $(0.124 \mathrm{~g}, 0.6 \mathrm{mmol})$ pinch by pinch for 2 minutes. The resulting mixture was stirred further for $12 \mathrm{~h}$. The dicyclohexyl urea (DCU) precipitates were filtered and the filtrated was diluted with $10 \mathrm{~mL}$ of DCM. The DCM layer was washed with saturated $\mathrm{NaHCO}_{3}$ solution $(2 \times 5 \mathrm{~mL})$ and water $(2 \times 5 \mathrm{~mL})$. The organic layer was dried over anhydr. $\mathrm{Na}_{2} \mathrm{SO}_{4}$ and concentrated under reduced pressure. The product obtained was further purified by column chromatography over silica gel with EtOAc/DCM as eluent. The final product was obtained as a white solid $(0.22 \mathrm{~g}$, Yield $60 \%)$, m.p. $45-46^{\circ} \mathrm{C}$. IR, $\tilde{v} / \mathrm{cm}^{-1}(\mathrm{KBr})$ : $1177,1644,1723,1645,1724,2854,2926,3313 .{ }^{1} \mathrm{H}$ NMR $\left(250 \mathrm{MHz}, \mathrm{CDCl}_{3}\right): \delta 0.75(\mathrm{~s}, 3 \mathrm{H}), 0.83-0.90$ $(\mathrm{m}, 6 \mathrm{H}), 1.25$ (br s, $22 \mathrm{H}), 1.28-2.16$ (steroidal $\mathrm{CH}$, $\mathrm{CH}_{2}$, alky $\left.\mathrm{CH} 2\right), 3.23(\mathrm{~m}, 1 \mathrm{H}), 4.71(\mathrm{~m}, 1 \mathrm{H}), 5.054(\mathrm{~d}$, $\mathrm{J}=2.5 \mathrm{~Hz}, 1 \mathrm{H}), 5.26(\mathrm{br} \mathrm{s}, 1 \mathrm{H}), 5.35(\mathrm{~m}, 1 \mathrm{H}) .{ }^{13} \mathrm{C} \mathrm{NMR}$ $\left(63 \mathrm{MHz}, \mathrm{CDCl}_{3}\right): \delta 12.16,14.07,17.60,22.33,22.66$, $22.79,25.57,26.91,27.19,28.58,29.28,29.33,29.53$, $29.57,29.63,29.67,31.35,31.47,31.90,32.77,33.53$, $34.29,34.47,34.53,34.85,37.76,39.53,40.83,42.97$, $45.04,47.38,70.69,73.73,76.49,160.50,172.95$.

\section{2c Compound 5: 3,12-Diformyl deoxycholic} acid $(0.25 \mathrm{~g}, 0.5 \mathrm{mmol}),{ }^{29}$ hexadecyalamine $(0.15$, $0.62 \mathrm{mmol}$ ), and DMAP $(6 \mathrm{mg}, 0.05 \mathrm{mmol})$ were dissolved in dry DCM. To this stirring mixture was added DCC $(0.124 \mathrm{~g}, 0.6 \mathrm{mmol})$ pinch by pinch for 2 minutes. The resulting mixture was stirred further for $12 \mathrm{~h}$. The DCU formed were filtered and the filtrated was diluted with $10 \mathrm{~mL}$ of DCM. The DCM layer was washed with saturated $\mathrm{NaHCO}_{3}(2 \times 5 \mathrm{~mL})$ and water $(2 \times 5 \mathrm{~mL})$. Dried over anhydr. $\mathrm{Na}_{2} \mathrm{SO}_{4}$ and concentrated under reduced pressure $\left(0.25 \mathrm{~g}\right.$, m.p. $\left.61-63^{\circ} \mathrm{C}\right)$. Then, the crude material was directly subjected to deformylation reaction by stirring the methanolic solution of triformyl amide over $\mathrm{K}_{2} \mathrm{CO}_{3}(0.40 \mathrm{~g}, 2.9 \mathrm{mmol})$ for $4-5 \mathrm{~h}$. After the reaction the suspension was filtered and the filtrate was concentrated under reduced pressure. The crude material was extracted into ethyl acetate $(15 \mathrm{~mL})$ and washed with saturated solution of $\mathrm{NaHCO}_{3}(2 \times$ $5 \mathrm{~mL})$ and water $(2 \times 5 \mathrm{~mL})$. The organic layer was dried over anhydr. $\mathrm{Na}_{2} \mathrm{SO}_{4}$ and concentrated under reduced pressure. The product obtained was further purified by column chromatography over silica gel with EtOH/DCM as eluent. The final product was obtained as a white solid (0.185 g, Yield 85\%), m.p.
85-87 C. Anal. Calcd. (\%) for $\mathrm{C}_{40} \mathrm{H}_{73} \mathrm{NO}_{3}+11 / 2 \mathrm{H}_{2} \mathrm{O}$ : $\mathrm{C}, 74.71 ; \mathrm{H}, 11.92 ; \mathrm{N}, 2.18$. found $\mathrm{C}-74.43, \mathrm{H}-11.95$, $\mathrm{N}-2.60$. IR, $\tilde{v} / \mathrm{cm}^{-1}$ (KBr): 1044, 1378, 1467, 1643, 2850, 2621, $3400 \mathrm{~cm}^{-1} .{ }^{1} \mathrm{H} \mathrm{NMR}\left(250 \mathrm{MHz}, \mathrm{CDCl}_{3}\right)$ : $\delta=0.679(\mathrm{~s}, 3 \mathrm{H}), 0.807-0.911(\mathrm{~m}, 6 \mathrm{H}), 0.981(\mathrm{~d}$, $\mathrm{J}=6.0 \mathrm{~Hz}, 3 \mathrm{H}), 1.02-1.19(\mathrm{~m}, 4 \mathrm{H}), 1.26(\mathrm{br} \mathrm{s}, 30 \mathrm{H})$, 1.3-1.9 (m, steroidal $\mathrm{CH}, \mathrm{CH}_{2}$ and alkyl), 3.21 (m, 2H), $3.59(\mathrm{~m}, 1 \mathrm{H}), 3.97$ (br s, 1H), $5.36(\mathrm{~m}, 1 \mathrm{H})$. LRMS (ESI): $\mathrm{m} / \mathrm{z}$ found 639.02 . HRMS (ESI): $\mathrm{m} / \mathrm{z}$ Calcd. for $\mathrm{C}_{40} \mathrm{H}_{73} \mathrm{NO}_{3} \mathrm{Na}$ 638.5488; found 638.5507.

\section{2d Compound 6: Hexadecyl 3,7,12-} triformylcholamide $(0.20,0.28 \mathrm{mmol})$ was stirred over $\mathrm{K}_{2} \mathrm{CO}_{3}(0.38,2.8 \mathrm{mmol})$ in $2.0 \mathrm{~mL} \mathrm{MeOH}$ for $4-5 \mathrm{~h}$. After the reaction the suspension was filtered and the filtrate was concentrated under reduced pressure. The crude material was extracted into ethyl acetate $(15 \mathrm{~mL})$ and washed with saturated solution of $\mathrm{NaHCO}_{3}(2 \times 5 \mathrm{~mL})$ and water $(2 \times 5 \mathrm{~mL})$. The organic layer was dried over anhydr. $\mathrm{Na}_{2} \mathrm{SO}_{4}$ and concentrated under reduced pressure. The product obtained was further purified by column chromatography over silica gel with EtOH/DCM as eluent. The final product was obtained as a white solid $(0.15 \mathrm{~g}$, Yield $85 \%)$, m.p. 81-83 ${ }^{\circ}$ C. Anal. Calcd. (\%) for $\mathrm{C}_{40} \mathrm{H}_{73} \mathrm{NO}_{4}+1 / 2 \mathrm{H}_{2} \mathrm{O}$ : C, 74.95; H, 11.64; N, 2.18; found C, 75.03; H, 11.65, $\mathrm{N}, 1.97 .{ }^{1} \mathrm{H}$ NMR $\left(250 \mathrm{MHz}, \mathrm{CDCl}_{3}\right): \delta 0.667(\mathrm{~s}, 3 \mathrm{H}$ Me-18), 0.84-0.87 (br s, triplet merged to singlet, $6 \mathrm{H}$ ), $0.982(\mathrm{~d}, \mathrm{~J}=5.75 \mathrm{~Hz}, 3 \mathrm{H}), 1.25(\mathrm{br} \mathrm{s}, 22 \mathrm{H}), 1.3-2.22$ (m, steroidal, $\mathrm{CH}, \mathrm{CH} 2$ and alkyl $\mathrm{CH} 2$ ), 2.84 (br s, 4H), $3.22(\mathrm{~m}, 2 \mathrm{H}), 3.43(\mathrm{~m}, 1 \mathrm{H}), 3.82(\mathrm{br} \mathrm{s}, 1 \mathrm{H}), 3.95$ (br s, $1 \mathrm{H}), 5.95(\mathrm{~m}, 1 \mathrm{H}) .{ }^{13} \mathrm{C}$ NMR $\left(63 \mathrm{MHz}, \mathrm{CDCl}_{3}\right): \delta$ $12.47,14.08,17.45,22.43,22.66,23.26,26.39,26.98$, 27.56, 28.15, 29.34, 29.59, 29.64, 29.69, 30.42, 31.74, $33.14,34.75,35.32$, 39.48, 39.58, 39.66, 41.54, 41.69, 46.40, 46.56, 68.48, 71.90, 73.12, 173.92. HRMS (ESI): $\mathrm{m} / \mathrm{z}$ Calcd. for $\mathrm{C}_{40} \mathrm{H}_{73} \mathrm{NO}_{4}+\mathrm{Na}$ : 655.5471; found 655.5519 .

2.2e Compound 7: 3,7,12-Triacetyl cholic acid $(1.00 \mathrm{~g}, 1.85 \mathrm{mmol}){ }^{30} \mathrm{DCC}(0.46 \mathrm{~g}, 2.22 \mathrm{mmol})$ and DMAP $(23 \mathrm{mg}, 0.186 \mathrm{mmol})$ were dissolved in dry DCM $(10 \mathrm{~mL})$. To this stirred mixture was added isopropylamine $(0.19 \mathrm{~mL}, 2.22 \mathrm{mmol})$ drop-wise for 5 minutes. The resulting mixture was stirred for additional $12 \mathrm{~h}$. The DCU formed were filtered and the filtrate was diluted with $10 \mathrm{~mL}$ of DCM. The DCM layer was washed with sat. $\mathrm{NaHCO}_{3}(2 \times 5 \mathrm{~mL})$ and water $(2 \times 5 \mathrm{~mL})$. The DCM layer was dried over anhydr. $\mathrm{Na}_{2} \mathrm{SO}_{4}$ and concentrated under reduced pressure. The crude material was further purified by column 
chromatography over silica gel using EtOAc/DCM as the eluent. The pure white product was obtained as a solid powder ( $0.58 \mathrm{~g}$, Yield 55\%), m.p. $92-93^{\circ} \mathrm{C}$, Anal. Calcd. (\%) for $\mathrm{C}_{33} \mathrm{H}_{53} \mathrm{NO}_{7}+1 \mathrm{H}_{2} \mathrm{O}$ : C-66.75, H- 9.33, $\mathrm{N}-2.35$; found C-66.67, H-9.15, N- 2.40. IR, $\tilde{v} / \mathrm{cm}^{-1}$ (KBr): 1024, 1248, 1377, 1537, 1647, 1736, 2872, 2943, 2965, 3315, 3385 $\mathrm{cm}^{-1}$. ${ }^{1} \mathrm{H}$ NMR $(250 \mathrm{MHz}$, $\left.\mathrm{CDCl}_{3}\right): \delta 0.700(\mathrm{~s}, 3 \mathrm{H} \mathrm{Me}-18), 0.792(\mathrm{~d}, \mathrm{~J}=6.25 \mathrm{~Hz}$, 3H Me-21), 0.888 (s, 3H Me-19), 1.20-1.99 (steroidal, $\left.\mathrm{CH}, \mathrm{CH}_{2}\right) 2.01(\mathrm{~s}, 3 \mathrm{H}), 2.05(\mathrm{~s}, 3 \mathrm{H}), 2.12(\mathrm{~s}, 3 \mathrm{H})$, $4.05(\mathrm{~m}, 1 \mathrm{H}), 4.55(\mathrm{~m}, 1 \mathrm{H}), 4.88(\mathrm{~d}, \mathrm{~J}=2.75 \mathrm{~Hz}, 1 \mathrm{H})$, 5.06 (br s, $1 \mathrm{H}), 5.278(\mathrm{~d}, \mathrm{~J}=7.75 \mathrm{~Hz}, 1 \mathrm{H}) .{ }^{13} \mathrm{C}$ NMR $\left(63 \mathrm{MHz} \mathrm{CDCl}_{3}\right): \delta 12.43,14.38,16.87,21.20,21.60$, 21.64, 21.77, 22.74, 22.97, 23.01, 25.77, 27.08, 27.43, $29.09,31.45,31.81,33.93,34.53,34.83,34.91,34.97$, $37.97,41.16,41.39,43.59,46.41,47.73,60.54,70.89$, $74.27,75.62,170.49,170.65,170.67,172.51$. HRMS (ESI): $\mathrm{m} / \mathrm{z}$ Calcd. for $\mathrm{C}_{33} \mathrm{H}_{53} \mathrm{NO}_{7}+\mathrm{Na}: 598.3687$; found 598.3675 .

2.2f Compound 8: A mixture containing iso-propyltriacetyl cholamide $(0.25 \mathrm{~g}, 0.43 \mathrm{mmol}), \mathrm{KOH}(0.25 \mathrm{~g}$, $4.5 \mathrm{mmol})$ and $\mathrm{MeOH}(2.0 \mathrm{~mL})$ were refluxed at $80^{\circ} \mathrm{C}$ for 8-10 h. After completion (monitored by TLC), the reaction mixture was cooled in ice bath and neutralized with dil. $\mathrm{HCl}$. The product was extracted into chloroform $(15 \mathrm{~mL})$ and washed with saturated solution of $\mathrm{NaHCO}_{3}(2 \times 5 \mathrm{~mL})$ and water $(2 \times 5 \mathrm{~mL})$, dried over anhydr. $\mathrm{Na}_{2} \mathrm{SO}_{4}$ and concentrated under reduced pressure. The product obtained was further purified by chromatography over silica gel with EtOH/DCM as eluent $(0.15 \mathrm{~g}$, Yield $75 \%)$, m.p. $242-244^{\circ} \mathrm{C}$. IR, $\tilde{v} / \mathrm{cm}^{-1}$ (KBr): 732, 1078, 1464, 1547, 1646, 2866, 2929, $3410,3432 \mathrm{~cm}^{-1}$. Anal. Calcd. (\%) for $\mathrm{C}_{27} \mathrm{H}_{47} \mathrm{NO}_{4}+2$ $1 / 2 \mathrm{H}_{2} \mathrm{O}: \mathrm{C}, 65.68 ; \mathrm{H}, 10.41 ; \mathrm{N}, 2.83$; found $\mathrm{C}$, 65.78; H, 9.70; N, 1.99. ${ }^{1} \mathrm{H}$ NMR $\left(250 \mathrm{MHz}, \mathrm{CDCl}_{3}\right)$ : $\delta 0.671$ (s, 3H Me-18), 0.876 (s, 3H Me-19), 0.984 (d, J =6.3 Hz Me-21), 1.129 (d, J =6.5 Hz, 6H), 1.262.30 (m, steroidal, $\mathrm{CH}, \mathrm{CH} 2$ ), 2.62 (br s, 4H). 3.43 $(\mathrm{m}, 1 \mathrm{H}), 3.823(\mathrm{~d}, \mathrm{~J}=2.25 \mathrm{~Hz}, 1 \mathrm{H}), 3.96(\mathrm{br} \mathrm{s}, 1 \mathrm{H})$, $4.07(\mathrm{~m}, 1 \mathrm{H}), 5.57(\mathrm{~d}, \mathrm{~J}=7.75 \mathrm{~Hz}, 1 \mathrm{H}) .{ }^{13} \mathrm{C}$ NMR $\left(63 \mathrm{MHz} \mathrm{CDCl}_{3}\right): \delta 12.71,17.70,22.68,22.99,23.47$, 26.66, 27.80, 28.43, 30.74, 31.92, 33.70, 34.97, 35.60, $35.61,39.73,39.89,41.39,41.75,41.98,46.68,46.96$, 68.67, 72.14, 73.30, 173.16. HRMS (ESI): m/z Calcd. for $\mathrm{C}_{27} \mathrm{H}_{47} \mathrm{NO}_{4}+\mathrm{Na}$ : 473.3436; found 473.3447.

$2.2 \mathrm{~g}$ Compound 10: Iso-Propyl cholamide $(0.27 \mathrm{~g}$, $0.60 \mathrm{mmol}), 12$-(1-pyrene) dodecanoic acid $(0.12 \mathrm{~g}$, $0.30 \mathrm{mmol})$ and DMAP $(4 \mathrm{mg}, 0.03 \mathrm{mmol})$ were mixed with dry DCM. To this stirring mixture was added DCC $(0.062 \mathrm{~g}, 0.3 \mathrm{mmol})$ pinch by pinch for few minutes. The resulting solution was stirred for $5 \mathrm{~h}$. DCU was removed by filtration and the filtrate was diluted with $10 \mathrm{~mL}$ of DCM. DCM layer was washed with saturated $\mathrm{NaHCO}_{3}$ solution $(2 \times 5 \mathrm{~mL})$ and water $(2 \times 5 \mathrm{~mL})$. The organic layer was dried over anhydr. $\mathrm{Na}_{2} \mathrm{SO}_{4}$ and concentrated under reduced pressure. The product obtained was further purified by column chromatography over silica gel with EtOAc/DCM as eluent. The final product was obtained as pale yellow foam $(0.052 \mathrm{~g}$, Yield $11 \%$ ), m.p. $86-87^{\circ} \mathrm{C}$. IR, $\tilde{v} / \mathrm{cm}^{-1}$ (KBr): 845,1182 , 1543, 1727, 2853, 2927, 3326, 3445. Anal. Calcd. (\%) for $\mathrm{C}_{55} \mathrm{H}_{77} \mathrm{NO}_{4}+1 \mathrm{H}_{2} \mathrm{O}: \mathrm{C}, 77.70 ; \mathrm{H}, 9.36 ; \mathrm{N}$, 1.65; found $\mathrm{C}, 77.70 ; \mathrm{H}, 9.06 ; \mathrm{N}, 1.99$. ${ }^{1} \mathrm{H}$ NMR $\left(250 \mathrm{MHz} \mathrm{CDCl}_{3}\right): \delta 0.67$ (s, 3H Me-18), $0.88(\mathrm{~s}$, $3 \mathrm{H} \mathrm{Me}-19), 0.97$ (d, J=6 Hz, 3H Me-21), 1.13 (d, $\mathrm{J}=6.5 \mathrm{~Hz}, 6 \mathrm{H}$ ), $1.22-2.33$ (steroidal $\mathrm{CH}, \mathrm{CH}_{2}$ and alkyl $\left.\mathrm{CH}_{2}\right), 3.27(\mathrm{t}, \mathrm{J}=7.5 \mathrm{~Hz}, 2 \mathrm{H}), 3.81$ (brs, $\left.1 \mathrm{H}\right)$, $3.95(\mathrm{br} \mathrm{s}, 1 \mathrm{H}), 4.08(\mathrm{~m}, 1 \mathrm{H}), 4.58(\mathrm{~m}, 1 \mathrm{H}), 5.35(\mathrm{~d}$, $\mathrm{J}=7.5 \mathrm{~Hz}, 1 \mathrm{H}), 7.86(\mathrm{~d}, \mathrm{~J}=8 \mathrm{~Hz}, 1 \mathrm{H}), 7.93-8.17(\mathrm{~m}$, $7 \mathrm{H}), 8.28(\mathrm{~d}, \mathrm{~J}=10 \mathrm{~Hz}) .{ }^{13} \mathrm{C}$ NMR $\left(63 \mathrm{MHz}, \mathrm{CDCl}_{3}\right)$ : $\delta \quad 12.73,14.40,17.66,21.23,22.67,23.00,23.37$, $25.15,25.28,25.84,26.88,26.96,27.72,28.49,29.37$, $29.46,29.63,29.78,30.02,31.79,32.14,33.67,33.81$, $34.16,34.66,34.89,35.05,35.12$, 35.46, 35.47, 39.69, $41.40,42.28,46.73,47.15,49.34,60.56,68.45,73.19$, $74.23,123.75,124.80,124.95,124.97,125.30,125.95$, $126.65,127.27,127.44,127.74,128.82,129.89$, 131.17, 131.67, 137.58, 172.93, 173.71. LRMS (ESI): $\mathrm{m} / \mathrm{z}$ Calcd. for $\mathrm{C}_{55} \mathrm{H}_{77} \mathrm{NO}_{4}+\mathrm{Na}$ : 854.57; found 854.40.

2.2h Compound 11: Iso-Propyl cholamide $(0.10 \mathrm{~g}$, $0.22 \mathrm{mmol}), \quad 12$-(1-pyrene)dodecanoic acid $(0.11 \mathrm{~g}$, $0.28 \mathrm{mmol}$ ) and DMAP (3 mg, $0.02 \mathrm{mmol}$ ) were mixed with dry DCM. To this stirring mixture DCC $(0.055 \mathrm{~g}$, $0.26 \mathrm{mmol}$ ) was added pinch by pinch for few minutes. The resulting solution was stirred for $12 \mathrm{~h}$. The precipitates of DCU was removed by filtration and the filtrate was diluted with $10 \mathrm{~mL}$ of DCM. DCM layer was washed with saturated $\mathrm{NaHCO}_{3}$ solution $(2 \times 5 \mathrm{~mL})$ and water $(2 \times 5 \mathrm{~mL})$. The organic layer was dried over anhydr. $\mathrm{Na}_{2} \mathrm{SO}_{4}$ and concentrated under reduced pressure. The product obtained was further purified by column chromatography over silica gel with EtOAc/DCM as eluent. The final product was obtained as pale yellow foam $(0.04 \mathrm{~g}$, Yield $15 \%)$, m.p. 70 71 ${ }^{\circ} \mathrm{C}$. Anal. Calcd. (\%) for $\mathrm{C}_{83} \mathrm{H}_{107} \mathrm{NO}_{6}+1 \mathrm{H}_{2} \mathrm{O}$ : C, $80.87 ; \mathrm{H}, 8.91 ; \mathrm{N}, 1.14$; found $\mathrm{C}, 80.67 ; \mathrm{H}, 9.59$; $\mathrm{N}, 1.12$. IR, $\tilde{v} / \mathrm{cm}^{-1}(\mathrm{KBr}): 845,1182,1543,1727$, 2853, 2927, 3385, 3417. ${ }^{1} \mathrm{H}$ NMR $\left(500 \mathrm{MHz} \mathrm{CDCl}_{3}\right)$ : 
$\delta 0.66$ (s, 3H Me-18), 0.89 (s, 3H Me-19), 0.96 (d, $\mathrm{J}=6.5 \mathrm{~Hz}, 3 \mathrm{H} \mathrm{Me}-21), 1.113(\mathrm{~d}, \mathrm{~J}=6.5 \mathrm{~Hz}, 6 \mathrm{H}), 1.14$ 2.11 (steroidal, $\left.\mathrm{CH}, \mathrm{CH}_{2}\right), 3.28-3.35(\mathrm{~m}, 4 \mathrm{H}), 3.95$ (br $\mathrm{s}, 1 \mathrm{H}), 4.05(\mathrm{~m}, 1 \mathrm{H}), 4.58(\mathrm{~m}, 1 \mathrm{H}), 4.885(\mathrm{~d}, \mathrm{~J}=2.5 \mathrm{~Hz}$, $1 \mathrm{H}), 5.14(\mathrm{~d}, \mathrm{~J}=7.5 \mathrm{~Hz}), 7.81-7.85(\mathrm{~m}, 2 \mathrm{H}), 7.93-$ $8.02(\mathrm{~m}, 6 \mathrm{H}), 8.05-8.10(\mathrm{~m}, 4 \mathrm{H}), 8.12-8.14(\mathrm{~m}, 4 \mathrm{H})$, 8.24-8.27 (m, 2H). ${ }^{13} \mathrm{C}$ NMR $\left(126 \mathrm{MHz}, \mathrm{CDCl}_{3}\right): \delta$ $12.73,14.41,17.72,21.23,22.72,23.02,23.24,24.50$, $24.90,25.16,25.25,25.32,25.54,25.74,25.96,26.65$, 26.94, 27.54, 28.37, 28.77, 29.37, 29.39, 29.58, 29.67, $29.73,29.79,29.81,29.84,29.86,30.02,30.06,31.06$, $31.58,31.85,32.13,32.16,32.98,33.80,34.00,34.18$, $34.57,34.99,35.02,35.17,35.33,38.38,41.17,41.40$, $42.37,46.79,47.50,49.37,60.57,70.77,78.01,73.98$, $74.31,123.73,124.80,124.95,124.97,125.30,125.31$, $125.93,126.65,127.27,127.42,127.73,128.83$, 129.90, 131.07, 131.68, 137.54, 173.31, 173.55. HRMS (ESI): $\mathrm{m} / \mathrm{z}$ Calcd. for $\mathrm{C}_{83} \mathrm{H}_{107} \mathrm{NO}_{6} \mathrm{Na}$ : 1236.7996 ; found 1236.8005 .

2.2i Compound 13: Hexadecyl cholamide $(0.15 \mathrm{~g}$, 0.24), 12-(1-pyrene)dodecanoic acid $\quad(0.12 \mathrm{~g}$, $0.30 \mathrm{mmol})$ and DMAP $(3.5 \mathrm{mg}, 0.03 \mathrm{mmol})$ were mixed with dry DCM $(1.0 \mathrm{~mL})$. To this stirred mixture was added DCC $(0.062 \mathrm{~g}, 0.3 \mathrm{mmol})$ pinch by pinch for few minutes. The resulting solution was stirred for $5 \mathrm{~h}$. DCU was removed by filtration and the filtrate was diluted with $10 \mathrm{~mL}$ of DCM. DCM layer was washed with saturated $\mathrm{NaHCO}_{3}$ solution $(2 \times 5 \mathrm{~mL})$ and water $(2 \times 5 \mathrm{~mL})$. The organic layer was dried over anhydr. $\mathrm{Na}_{2} \mathrm{SO}_{4}$ and concentrated under reduced pressure. The product obtained was further purified by column chromatography over silica gel with EtOAc/DCM as eluent. The final product was obtained as pale yellow foam $(0.12 \mathrm{~g}$, Yield $50 \%)$, m.p. $54-55^{\circ} \mathrm{C}$. Anal. Calcd. (\%) for $\mathrm{C}_{68} \mathrm{H}_{103} \mathrm{NO}_{5}+11 / 2 \mathrm{H}_{2} \mathrm{O}: \mathrm{C}, 78.41 ; \mathrm{H}$, 10.25; N, 1.34; found $\mathrm{C}, 78.18, \mathrm{H}, 9.80 ; \mathrm{N}, 0.92$. IR, $\tilde{v} / \mathrm{cm}^{-1}(\mathrm{KBr}): 844,1465,1547,1645,1728,2853$, 2924, 3324, 3384, 3419. ${ }^{1} \mathrm{H}$ NMR $\left.(500 \mathrm{MHz} \mathrm{CDCl})_{3}\right): \delta$ 0.68 (s, 3H), 0.86-0.89 (m, 6H), 1.004-1.13 (m, 6H), 1.25-1.27 (br peak, 42 H), 1.34-2.35 (steroidal $\mathrm{CH}$, $\mathrm{CH}_{2}$, alkylCH $\mathrm{CH}_{2}$ ), 3.24 (br s, $\left.2 \mathrm{H}\right), 3.33$ (t, J $\left.=8 \mathrm{~Hz}, 2 \mathrm{H}\right)$, $4.57(\mathrm{~m}, 1 \mathrm{H}), 7.87(\mathrm{~d}, \mathrm{~J}=7.5 \mathrm{~Hz}), 7.97-8.16(\mathrm{~m}, 7 \mathrm{H})$, $8.282(\mathrm{~d}, \mathrm{~J}=9 \mathrm{~Hz}, 1 \mathrm{H}) .{ }^{13} \mathrm{C}$ NMR $\left(126 \mathrm{MHz}, \mathrm{CDCl}_{3}\right): \delta$ $12.43,14.08,17.65,22.39,22.67,23.26,25.08,25.37$, $25.71,26.61,26.84,26.92,27.52,28.17,29.19,29.27$, $29.34,29.43,29.57,29.59,29.64,29.65,29.69,29.813$, $31.91,31.93,33.60,34.53,34.68,34.86,34.90,35.19$, $35.20,39.34,40.03,41.22,42.12,46.44,68.33,73.10$, $73.99,123.53,124.59,124.74,124.76,125.10,125.72$, $126.45,127.06,127.53,128.62,129.69,130.96$,
131.46, 137.36, 173.43. HRMS (ESI): m/z Calcd. for $\mathrm{C}_{68} \mathrm{H}_{103} \mathrm{NO}_{5}+\mathrm{Na}$ : 1036.7734; found 1036.7737.

2.2j Compound 14: Hexadecyl deoxycholamide $(0.20 \mathrm{~g}, 0.33 \mathrm{mmol}), 4-(1-$ pyrene $)$ butyric acid $(0.112 \mathrm{~g}$, $0.39 \mathrm{mmol})$ and DMAP $(6 \mathrm{mg}, 0.05 \mathrm{mmol})$ were mixed with dry DCM. To this stirring mixture DCC $(0.083 \mathrm{~g}$, $0.4 \mathrm{mmol}$ ) was added pinch by pinch for $5 \mathrm{~min}$. The resulting solution was stirred for $5 \mathrm{~h}$. DCU was removed by filtration and the filtrate was diluted with $10 \mathrm{~mL}$ of DCM. DCM layer was washed with saturated $\mathrm{NaHCO}_{3}$ solution $(2 \times 5 \mathrm{~mL})$ and water $(2 \times 5 \mathrm{~mL})$. The organic layer was dried over anhydr. $\mathrm{Na}_{2} \mathrm{SO}_{4}$ and concentrated under reduced pressure. The product obtained was further purified by chromatography over silica gel with EtOAc/DCM as eluent. The final product was obtained as pale yellow foam $(0.17 \mathrm{~g}$, Yield $60 \%)$, m.p. $65-68^{\circ} \mathrm{C}$. IR, $\tilde{v} / \mathrm{cm}^{-1}(\mathrm{KBr}): 844,1383,1645$, 1728, 2853, 2924, 3440. ${ }^{1} \mathrm{H}$ NMR $\left(250 \mathrm{MHz} \mathrm{CDCl}_{3}\right)$ : $\delta 0.65(\mathrm{~s}, 3 \mathrm{H}), 0.86-0.95(\mathrm{~m}, 6 \mathrm{H}), 0.96(\mathrm{~d}, \mathrm{~J}=6 \mathrm{~Hz})$, $1.02-1.1 .20(\mathrm{~m}, 3 \mathrm{H}), 1.26$ (br s, 30H), 1.30-2.50 (steroidal $\mathrm{CH}, \mathrm{CH}_{2}$ and alkyl $\left.\mathrm{CH}_{2}\right), 3.22(\mathrm{~m}, 2 \mathrm{H}), 3.38$ $(\mathrm{t}, \mathrm{J}=8 \mathrm{~Hz}, 2 \mathrm{H}), 3.94(\mathrm{br} \mathrm{s}, 1 \mathrm{H}), 4.75(\mathrm{~m}, 1 \mathrm{H}), 5.46(\mathrm{~m}$, $1 \mathrm{H}), 7.86(\mathrm{~d}, \mathrm{~J}=8 \mathrm{~Hz}, 1 \mathrm{H}), 7.94-8.18(\mathrm{~m}, 7 \mathrm{H}), 8.31$ $(\mathrm{d}, \mathrm{J}=9.25 \mathrm{~Hz}, 1 \mathrm{H}) \cdot{ }^{13} \mathrm{C}$ NMR $\left(63 \mathrm{MHz}, \mathrm{CDCl}_{3}\right): \delta$ $12.93,14.29,17.63,22.87,23.28,23.77,26.20,26.78$, $27.13,27.19,27.65,28.92,29.51,29.54,29.75,29.79$, $29.89,31.93,32.11,32.45,33.04,33.79,33.86,34.30$, $34.62,35.09,35.34,36.16,39.76,42.10,46.67,47.49$, $48.48,73.28,74.46,123.56,124.91,125.01,125.05$, $125.20,125.28,125.98,126.85,127.57,127.68$, $128.94,130.15,131.12,131.62,136.02,173.17$, 173.63. LRMS (ESI): $\mathrm{m} / \mathrm{z}$ found 908.76. HRMS (ESI): $\mathrm{m} / \mathrm{z}$ Calcd. for $\mathrm{C}_{60} \mathrm{H}_{87} \mathrm{NO}_{4}+\mathrm{Na}$ : 908.6533; found 908.6570.

2.2k Compound 15: Hexadecyl cholamide $(0.12 \mathrm{~g}$, $0.195 \mathrm{mmol}), 12$-(1-pyrene) dodecanoic acid $(0.1 \mathrm{~g}$, $0.25 \mathrm{mmol})$ and DMAP $(2.5 \mathrm{mg}, 0.02 \mathrm{mmol})$ were mixed with dry DCM. To this stirring mixture DCC was added pinch by pinch for few minutes. The resulting solution was stirred for $5 \mathrm{~h}$. DCU was removed by filtration and the filtrate was diluted with $10 \mathrm{~mL}$ of DCM. DCM layer was washed with saturated $\mathrm{NaHCO}_{3}$ solution $(2 \times 5 \mathrm{~mL})$ and water $(2 \times 5 \mathrm{~mL})$. The organic layer was dried over anhydr. $\mathrm{Na}_{2} \mathrm{SO}_{4}$ and concentrated under reduced pressure. The product obtained was further purified by column chromatography over silica gel with EtOAc/DCM as eluent. The final product was obtained as pale yellow foam $(0.11 \mathrm{~g}$, Yield $55 \%)$, m.p. $52-54^{\circ} \mathrm{C}$. Anal. Calcd. (\%) 
for $\mathrm{C}_{68} \mathrm{H}_{103} \mathrm{NO}_{4}+2 \mathrm{H}_{2} \mathrm{O}: \mathrm{C}, 78.94 ; \mathrm{H}, 10.42 ; \mathrm{N}, 1.37$; found $\mathrm{C}, 78.55 ; \mathrm{H}, 10.02 ; \mathrm{N}, 1.92$. IR, $\tilde{v} / \mathrm{cm}^{-1}(\mathrm{KBr})$ : $844,1181,1465,1643,1731,2852,2924.3380 .{ }^{1} \mathrm{H}$ NMR $\left(250 \mathrm{MHz} \mathrm{CDCl}_{3}\right): \delta 0.57(\mathrm{~s}, 3 \mathrm{H}), 0.77(\mathrm{~s}, 3 \mathrm{H})$, $0.92(\mathrm{t}, \mathrm{J}=7 \mathrm{~Hz}, 3 \mathrm{H}), 1.01(\mathrm{~d}, \mathrm{~J}=6 \mathrm{~Hz}, 3 \mathrm{H}), 1.26-$ 2.01 (m, steroidal, alkyl $\left.\mathrm{CH}, \mathrm{CH}_{2}\right), 3.06-3.25(\mathrm{~m}, 4 \mathrm{H})$, $3.81(\mathrm{br} \mathrm{s}, 1 \mathrm{H}), 4.79(\mathrm{t}, \mathrm{J}=6.25 \mathrm{~Hz}, 1 \mathrm{H}), 4.97(\mathrm{~m}, 1 \mathrm{H})$, 7.72-8.01 (m, 8H), $8.23(\mathrm{~d}, \mathrm{~J}=9.25,1 \mathrm{H}) .{ }^{13} \mathrm{C}-\mathrm{NMR}$ $\left(\mathrm{C}_{6} \mathrm{D}_{6}, 63 \mathrm{MHz}\right): \delta 13.33,14.71,17.99,23.46,23.60$, $24.31,25.75,25.88,26.72,26.89,27.55,27.70,29.51$, $29.91,30.05,30.18,30.24,30.35,30.49,30.56,30.69$, $31.60,32.51,32.61,32.69,33.22,33.30,33.93,34.25$, $34.62,35.32$, 35.61, 35.98, 36.62, 39.98, 42.52, 47.14, $47.72,48.71,73.29,74.48,124.22,125.39,125.56$, $125.60,126.15,126.20,126.41,127.28,129.57$, 130.71, 131.89, 132.40, 137.83, 172.73, 173.07. LRMS (ESI): $\mathrm{m} / \mathrm{z}$ found 1020.90. HRMS (ESI): $\mathrm{m} / \mathrm{z}$ Calcd. $\mathrm{C}_{68} \mathrm{H}_{103} \mathrm{NO}_{4} \mathrm{Na} 1022.7852$; found 1022.7860 .

2.21 Compound $15+$ Compound 24 (TNF): Compound $15(5.5 \mathrm{mM})$ and TNF $(5.5 \mathrm{mM})$ were dissolved in benzene $\left[\mathrm{D}_{6}\right]$ and NMR spectrum was recorded. ${ }^{1} \mathrm{H}$ NMR $\left(250 \mathrm{MHz}, \mathrm{C}_{6} \mathrm{D}_{6}\right): \delta 0.59(\mathrm{~s}, 3 \mathrm{H}), 0.79(\mathrm{~s}, 3 \mathrm{H})$, $0.92(\mathrm{t}, \mathrm{J}=6.75 \mathrm{~Hz}, 3 \mathrm{H}), 1.019(\mathrm{~d}, \mathrm{~J}=6 \mathrm{~Hz}, 3 \mathrm{H}), 1.17-$ 2.06 (m, steroidal, alkyl, $\mathrm{CH}, \mathrm{CH}_{2}$ ), $2.28(\mathrm{t}, \mathrm{J}=7.5 \mathrm{~Hz}$, $2 \mathrm{H}), 3.04-3.19(\mathrm{~m}, 4 \mathrm{H}), 3.83(\mathrm{br} \mathrm{s}, 1 \mathrm{H}), 4.82(\mathrm{t}$, $\mathrm{J}=6 \mathrm{~Hz}, 1 \mathrm{H}), 4.95(\mathrm{~m}, 1 \mathrm{H}), 7.32(\mathrm{~d}, \mathrm{~J}=8.5 \mathrm{~Hz}, 1 \mathrm{H})$, 7.42-7.67 (m, 8H), 7.902 (d, J =2 Hz,), 7.945 (br s, $0.5 \mathrm{H}), 8.05-8.07(\mathrm{~m}, 2 \mathrm{H}) .{ }^{13} \mathrm{C} \mathrm{NMR}\left(126 \mathrm{MHz}, \mathrm{C}_{6} \mathrm{D}_{6}\right)$ : $\delta 13.35,14.73,18.00,23.48,23.63,24.34,25.44,25.91$, $26.75,27.22,27.56,27.73,28.27,29.54,29.95,30.09$, $30.20,30.28,30.38,30.51,30.58,30.70,31.62,32.30$, $32.53,32.70,33.23,33.30,34.03,34.10,34.27,34.66$, $35.34,35.64,36.03,36.65,40.00,42.56,47.17,47.77$, $48.75,73.33,74.51,119.45,122.01,124.08,125.07$, $125.27,125.34,125.49,125.61,126.51,127.10$, $127.44,127.69,127.82,130.08,130.11,131.31$, $131.78,136.06,137.83,137.95,138.67,142.88$, 144.46, 148.81, 150.09, 172.77, 173.13, 185.41.

$2.2 \mathrm{~m}$ TNF (Compound 24) ${ }^{1} \mathrm{H}-\mathrm{NMR} \quad(500 \mathrm{MHz}$, $\left.C_{6} D_{6}\right): \delta 7.41(\mathrm{~d}, \mathrm{~J}=8.5 \mathrm{~Hz}, 1 \mathrm{H}), 7.63(\mathrm{~d}, \mathrm{~J}=2 \mathrm{~Hz}$, $0.5 \mathrm{H}), 7.64(\mathrm{~d}, \mathrm{~J}=2.5 \mathrm{~Hz}, 0.5 \mathrm{H}), 8.05(\mathrm{~d}, \mathrm{~J}=2.5 \mathrm{~Hz}$, $1 \mathrm{H}), 8.11(\mathrm{~d}, \mathrm{~J}=2 \mathrm{~Hz}, 1 \mathrm{H}), 8.16(\mathrm{~d}, \mathrm{~J}=2.5 \mathrm{~Hz}, 1 \mathrm{H}) .{ }^{13} \mathrm{C}$ NMR $\left(126 \mathrm{MHz}, \mathrm{C}_{6} \mathrm{D}_{6}\right): \delta 119.79,122.35,125.24$, $127.53,129.07,129.10,130.38,136.27,138.13$, $138.91,143.11,144.82,149.18,150.33,185.69$, 208.46. 2.2n Compound 16: The starting compound, dodecyl deoxycholate, was prepared by reacting deoxycholic acid $(0.5 \mathrm{~g}, 1.27 \mathrm{mmol})$ and 1-dodecylbromide $(0.375 \mathrm{~g}$, $1.5 \mathrm{mmol})$ in the presence of DBU $(0.225 \mathrm{~g}, 1.48 \mathrm{mmol})$ in $3 \mathrm{~mL} \mathrm{DMF}$ as the solvent. The reaction mixture was stirred at $60^{\circ} \mathrm{C}$ for $12 \mathrm{~h}$. After the reaction, the reaction mixture was poured into a beaker containing dil. $\mathrm{HCl}$ solution $(20 \mathrm{~mL})$ and the procuct was extracted with DCM $(20 \mathrm{~mL} \times 2)$. The organic layer was washed with dil. $\mathrm{HCl}(10 \mathrm{~mL} \times 2)$, sat. $\mathrm{NaHCO}_{3}$ solution $(10 \mathrm{~mL} \times 2)$, followed by water wash $(10 \mathrm{~mL} \times 2)$. The solvent was removed under reduced pressure and the product was dried under high vacuum. The yeild $(0.66 \mathrm{~g}, 93 \%)$ of the reaction was nearly quantitative. Then 1-pyrene butyric acid $(0.25 \mathrm{~g}, 0.87 \mathrm{mmol})$ and dodecyldeoxycholate in dry DCM was stirred at $25^{\circ} \mathrm{C}$ in the presence of DCC $(0.186 \mathrm{~g}, 0.9 \mathrm{mmol})$ and a catalytic amount of DMAP $(0.01 \mathrm{~g}, 0.08 \mathrm{mmol})$ for $24 \mathrm{~h}$. The DCU formed was filtered. The filtrate was washed with sat. $\mathrm{NaHCO}_{3}$ solution $(10 \mathrm{~mL})$, followed by water wash $(10 \mathrm{~mL})$. The organic layer was concentrated after drying over anhydr. $\mathrm{Na}_{2} \mathrm{SO}_{4}$. The product was chromatographed on a silica gel column using EtOAc/DCM as the eluent $(0.21 \mathrm{~g}$, Yield 30\%), Anal. Calcd. (\%) for $\mathrm{C}_{55} \mathrm{H}_{76} \mathrm{O}_{5}+\mathrm{H}_{2} \mathrm{O}: \mathrm{C}, 79.09 ; \mathrm{H}, 9.41$; found $\mathrm{C}, 78.78 ; \mathrm{H}$, 9.43. IR, $\tilde{v} / \mathrm{cm}^{-1}$ (KBr): 828, 1181, 1466, 1639, 1733, 2850, 2928. 3040. ${ }^{1} \mathrm{H}$ NMR $\left(300 \mathrm{MHz} \mathrm{CDCl}_{3}\right): \delta 0.66$ (s, 3H), 0.85-0.93 (m, 6H), $0.97(\mathrm{~d}, \mathrm{~J}=6 \mathrm{~Hz}, 3 \mathrm{H}), 1.2$ 2.5 (steroidal $\mathrm{CH}, \mathrm{CH}_{2}$, alkyl $\left.\mathrm{CH}_{2}\right), 3.96$ (br s, $1 \mathrm{H}$ ), $4.05(\mathrm{t}, \mathrm{J}=6.6 \mathrm{~Hz}, 3 \mathrm{H}), 4.75(\mathrm{~m}, 1 \mathrm{H}), 7.85(\mathrm{~d}, 7.8 \mathrm{~Hz}$, $1 \mathrm{H}), 7.94-8.08(\mathrm{~m}, 3 \mathrm{H}), 8.08-8.20(\mathrm{~m}, 4 \mathrm{H}), 8.31$ (d, $9.3 \mathrm{~Hz}, 1 \mathrm{H}) \cdot{ }^{13} \mathrm{C}$ NMR $\left(75 \mathrm{MHz}, \mathrm{CDCl}_{3}\right): \delta 12.88$, 14.26, 17.42, 22.83, 23.29, 23.86, 26.12, 26.31, 26.80, $27.33,27.66,28.83,29.42,29.49,29.80,30.59,31.13$, $31.59,32.07,32.50,33.05,33.78,34.29,35.36,35.46$, $36.22,36.61,42.29,46.66,47,45,48.37,64.59,71.81$, $73.25,123.60,124.91,125.05,125.22,125.35,126.00$, $126.88,127.61,127.66,128.99,130.17,131.19$, 131.70, 131.67, 136.08, 173.19, 174.50. LRMS (ESI): $\mathrm{m} / \mathrm{z}$ found 841. HRMS (ESI): $\mathrm{m} / \mathrm{z}$ Calcd. $\mathrm{C}_{55} \mathrm{H}_{76} \mathrm{O}_{5} \mathrm{Na}$ 840.1952; found 840.1947.

2.2o Compound 17: 1-Pyrene butyric acid $(0.25 \mathrm{~g}$, $0.87 \mathrm{mmol}$ ) was dispersed in $2.5 \mathrm{~mL}$ dry DCM. To this dispersion was added oxalyl chloride $(0.13 \mathrm{~g}$, $1.02 \mathrm{mmol})$, a catalytic amount of DMF $(5 \mu \mathrm{L})$. The mixture was stirred for $2 \mathrm{~h}$ at $25^{\circ} \mathrm{C}$. The solvents were removed using a $\mathrm{KOH}$ trap connected between the vacuum pump and the sample. The acid chloride so obtained was dissolved in $1 \mathrm{~mL}$ of dry DCM 
and added to a mixture containing methyl deoxycholate $(0.3 \mathrm{~g}, 0.74 \mathrm{mmol})$ and dry pyridine $(2 \mathrm{~mL})$ drop by drop for $30 \mathrm{~min}$. The mixture was stirred for $2 \mathrm{~h}$. After the reaction, the mixture was quenched with $10 \mathrm{~mL}$ of dil.HCl. The product was extracted with DCM $(20 \mathrm{~mL} \times 2)$ and washed with dil. $\mathrm{HCl}$ $(10 \mathrm{~mL})$, sat. $\mathrm{NaHCO}_{3}$ solution $(10 \mathrm{~mL})$, followed by water wash $(10 \mathrm{~mL})$. The organic layer was concentrated after drying over anhydr. $\mathrm{Na}_{2} \mathrm{SO}_{4}$. The product was chromatographed on a silica gel column using EtOAc/DCM as the eluent (0.08 g, Yield 14\%), Anal. Calcd. (\%) for $\mathrm{C}_{44} \mathrm{H}_{54} \mathrm{O}_{5}+\mathrm{H}_{2} \mathrm{O}$ : C, 77.61; H, 8.29; found $\mathrm{C}, 77.42 ; \mathrm{H}, 7.99$. IR, $\tilde{v} / \mathrm{cm}^{-1}(\mathrm{KBr})$ : 832, 1185, 1458, 1649, 1730, 2849, 2928. 3046. ${ }^{1} \mathrm{H}$ NMR $\left(300 \mathrm{MHz} \mathrm{CDCl}_{3}\right): \delta 0.67(\mathrm{~s}, 3 \mathrm{H}), 0.91(\mathrm{~s}, 3 \mathrm{H}), 0.97$ $(\mathrm{d}, \mathrm{J}=5.7 \mathrm{~Hz}, 3 \mathrm{H}), 1.0-2.5\left(\mathrm{~m}\right.$, steroidal $\left.\mathrm{CH}, \mathrm{CH}_{2}\right)$, $3.39(\mathrm{t}, \mathrm{J}=7.2 \mathrm{~Hz}, 2 \mathrm{H}), 3.67(\mathrm{~s}, 3 \mathrm{H}), 3.96(\mathrm{br} \mathrm{s}, 1 \mathrm{H})$, $4.79(\mathrm{~m}, 1 \mathrm{H}), 7.86(\mathrm{~d}, 7.8 \mathrm{~Hz}, 1 \mathrm{H}), 7.97(\mathrm{~d}, 7.8 \mathrm{~Hz}, 1 \mathrm{H})$, 8.01-8.07 (m, 2H), 8.09-8.21 (m, 4H), $8.32(\mathrm{~d}, 9.3 \mathrm{~Hz}$, $1 \mathrm{H}) .{ }^{13} \mathrm{C}$ NMR $\left(75 \mathrm{MHz}, \mathrm{CDCl}_{3}\right): \delta 12.93,17.53$, $23.31,23.80,26.22,26.82,27.16,27.21,27.58,28.95$, $31.12,31.27,32.47,33.05,33.90,34.35,34.64,35.12$, 35.25, 36.20, 42.11,46.72, 47.57, 48.51, 51.67, 73.31, $74.45,123.59,124.93,125.05,125.23,125.33,125.99$, $126.88,127.60,127.68,128.98,130.18,131.17$, 131.69, 131.66, 136.09, 173.20, 174.94. LRMS (ESI): $\mathrm{m} / \mathrm{z}$ found 686. HRMS (ESI): $\mathrm{m} / \mathrm{z}$ Calcd. $\mathrm{C}_{44} \mathrm{H}_{54} \mathrm{O}_{5} \mathrm{Na}$ 685.8995; found 685.8989 .

2.2p Compound 23: 1-Pyrene butyric acid $(0.25 \mathrm{~g}$, $0.87 \mathrm{mmol}$ ) was dissolved in $2 \mathrm{~mL}$ DMF. To this clear solution DBU $(0.152 \mathrm{~g}, 0.99 \mathrm{mmol})$ was added dropwise and stirred for $15 \mathrm{~min}$. 1-Dodecyl bromide $(0.25$, $1.0 \mathrm{mmol}$ ) was added to above solution maintained at $60^{\circ} \mathrm{C}$. The resulting mixture was stirred for additional $6 \mathrm{~h}$. The progress of the reaction was monitored by TLC. After the disappearance of the starting material, DMF was distilled off under reduced pressure. The residue was extracted into DCM $(25 \mathrm{~mL} \times 2)$. The organic layer was washed with dil. $\mathrm{HCl}(10 \mathrm{~mL} \times$ 2) followed by water $(10 \mathrm{~mL})$ and dried over anhydr. $\mathrm{Na}_{2} \mathrm{SO}_{4}$. The crude material was obtained after the solvent removal was further purified over silica gel column using DCM/PE as the eluent. The final product was isolated as pale yellow oil $(0.32 \mathrm{~g}$, Yield $84 \%)$, Anal. Calcd. (\%) for $\mathrm{C}_{31} \mathrm{H}_{38} \mathrm{O}_{2}: \mathrm{C}, 84.12 ; \mathrm{H}, 8.65$; found $\mathrm{C}, 78.78 ; \mathrm{H}, 9.43$. IR, $\tilde{v} / \mathrm{cm}^{-1}$ (KBr): 789, 821, 1211, 1458, 1649, 1741, 2849, 2925. 3048. ${ }^{1} \mathrm{H}$ NMR $\left(300 \mathrm{MHz} \mathrm{CDCl}_{3}\right): \delta 0.85(\mathrm{t}, \mathrm{J}=6.5 \mathrm{~Hz}, 3 \mathrm{H}), 1.18-$ $1.35(\mathrm{~m}, 20 \mathrm{H}), 2.17(\mathrm{~m}, 2 \mathrm{H}), 2.35(\mathrm{t}, \mathrm{J}=5 \mathrm{~Hz}, 2 \mathrm{H})$, $3.38(\mathrm{t}, \mathrm{J}=7 \mathrm{~Hz}, 2 \mathrm{H}), 3.65(\mathrm{t}, \mathrm{J}=6 \mathrm{~Hz}, 2 \mathrm{H}), 7.85(\mathrm{~d}$,
$7.8 \mathrm{~Hz}, 1 \mathrm{H}), 7.96(\mathrm{~d}, 7.8 \mathrm{~Hz}, 1 \mathrm{H}), 8.00-8.05(\mathrm{~m}, 2 \mathrm{H})$, $8.07-8.19(\mathrm{~m}, 4 \mathrm{H}), 8.30(\mathrm{~d}, 9.25 \mathrm{~Hz}, 1 \mathrm{H}) .{ }^{13} \mathrm{C}$ NMR $\left(75 \mathrm{MHz}, \mathrm{CDCl}_{3}\right): \delta 14.26,22.83,26.12,26.51,29.42$, $29.49,29.80,30.59,32.07,32.64,33.22,35.46,36.61$, $64.59,123.63,124.89,125.01,125.23,125.33,125.97$, $126.89,127.63,127.69,128.97,130.15,131.17$, 131.68, 131.71, 136.06, 173.22. LRMS (ESI): $\mathrm{m} / \mathrm{z}$ found 466. HRMS (ESI): m/z Calcd. for $\mathrm{C}_{31} \mathrm{H}_{38} \mathrm{O}_{2} \mathrm{Na}$ 465.6313; found 465.6320 .

\section{Results and discussion}

The synthesis of bile acid derivatives 4-8, pyrene bile acid conjugates 10, 11, 13-17 and pyrene derivative 23 was achieved using common organic synthesis techniques (chart 1 , see experimental section for details) and the gelation tests were performed in a set of different organic solvents.

To our disappointment the compounds 9-18 either remained in solution or precipitated within $12 \mathrm{~h}$ of testing and no sign of gelation was detected. However, an intense red coloured charge transfer (CT) complex was formed when trinitrofluorenone, TNF (24, chart 1) was added into solutions of 9-18. The gelation tests were made at $1: 1 \% \mathrm{w} / \mathrm{v}$ of the gelator and TNF in an organic solvent. The gelation was visually confirmed by turning the test tube upside down and monitoring the flow of the gel. The observations were carried out at room temperature $\left(25^{\circ} \mathrm{C}\right)$ in organic solvent listed in table 1 .

\subsection{Gelation studies}

We have introduced a few pyrene analogues and bile acid analogues as potent gelators of organic solvents. As both pyrene $e^{25 b}$ and bile acid ${ }^{25 c}$ moieties are known as potent structural fragments in gelling these moieties were first tested separately in gelation. No gelation was seen with the simplest pyrene molecule 1-pyrene carboxylic acid (19) or its mixture with 1-aminopyrene (20) (prepared by heating the 1:1 mixture of the amine and acid in an organic solvent during the test), even in the presence of an equivalent amount of CT agent, TNF. Similarly, no gelation was seen with the 1:1 conjugate of deoxycholic acid or cholic acid and 1-aminopyrene in the presence of an equivalent amount of TNF. The mixture precipitated out in most of the organic solvents used for the study. PBA alone (21) or in the presence of TNF does not gel organic solvents. Both 21 and 21+TNF remain in solution when mixed in benzene, toluene and chlorobenzene. However, when 1-decanol or cyclohexanol was used, a gelatinous precipitate, yet 

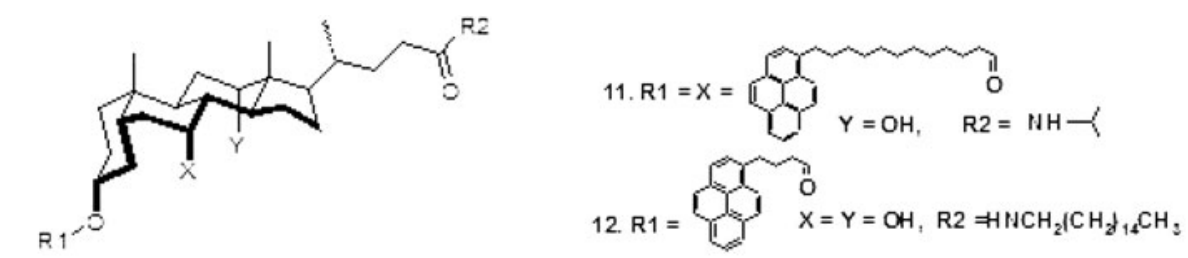

1. R1 $=\mathrm{C}\left(\mathrm{O} \mathrm{CH}_{3} \mathrm{X}=\mathrm{Y}=\mathrm{OC}(\mathrm{O}) \mathrm{CH}_{3} \mathrm{R} 2=\mathrm{OH}\right.$

2. R1 $=\mathrm{C} \mid \mathrm{O}$ ㅇ $\mathrm{H} X=\mathrm{Y}=\mathrm{OC} \mid \mathrm{O} \mathrm{H}, \mathrm{R} 2=\mathrm{OH}$

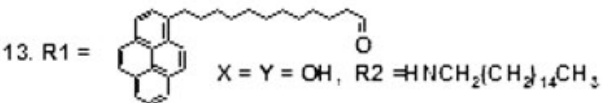

3. R1 $=\mathrm{ClO}$ 아 $\mathrm{CH}_{3} \mathrm{X}=\mathrm{Y}=\mathrm{OC}(\mathrm{O}) \mathrm{CH}_{3} \mathrm{R} 2=\mathrm{OH}$

4. R1 $\left.=\mathrm{C}(\mathrm{O}) \mathrm{H}, \mathrm{X}=\mathrm{H}, \mathrm{Y}=\mathrm{OC}(\mathrm{O}) \mathrm{H}, \mathrm{R} 2=\mathrm{HNCH}_{2} \mathrm{CH}_{2}\right\}_{14} \mathrm{CH}_{3}$

5. R1 $=\mathrm{H}, \mathrm{X}=\mathrm{H}, \mathrm{Y}=\mathrm{OH}, \mathrm{R} 2=\mathrm{HNCH}_{2}\left|\mathrm{CH}_{2}\right|_{14} \mathrm{CH}_{3}$

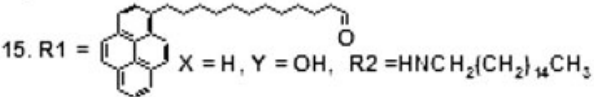

6. $\mathrm{R} 1=\mathrm{H}, \mathrm{X}=\mathrm{Y}=\mathrm{OH}, \mathrm{R} 2=\mathrm{HNCH} \mathrm{CH}_{2} \mathrm{CH}_{14} \mathrm{CH}_{2}$

7. R1 $=\mathrm{C}_{1} \mathrm{O} \mathrm{CH}_{2} \mathrm{X}=\mathrm{Y}=\mathrm{OC}_{1} \mathrm{O}_{\mathrm{CH}_{2}} \mathrm{R2}=\mathrm{NH}-\mathrm{C}$

8. R1 $=H, X=Y=O H R 2=N H-$
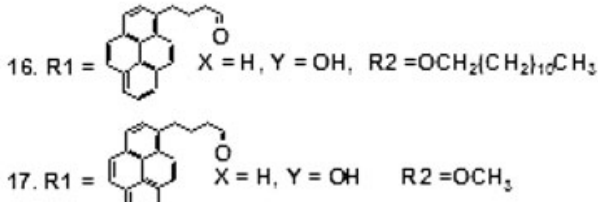

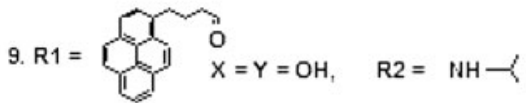

18. $R 1=H, X=H, Y=O H, R 2=$

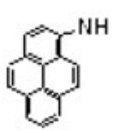

10.R1 $=$<smiles>N#[N+]c1cc2c(c([N+](=O)[O-])c1)-c1ccc([N+](=O)[O-])cc1C2=O</smiles><smiles>[R]OC(=O)CCCc1ccc2ccc3cccc4ccc1c2c34</smiles><smiles>Oc1ccc2ccc3cccc4ccc1c2c34</smiles>

23. $\mathrm{R}=\mathrm{CH}_{2}\left(\mathrm{CH}_{2}\right)_{10} \mathrm{CH}$,

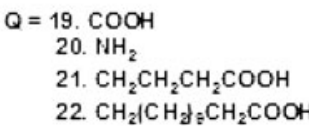

Chart 1. Gelator molecules used in the present study.

not a gel, was observed. This finding is interesting since when a longer alkyl chain used, viz., compound $\mathbf{2 2}$, strong CT gels in many organic solvents are formed as shown by us previously. ${ }^{24}$

Interestingly, PBA esters of bile acid conjugates (esterification of $3 \alpha-\mathrm{OH}$ ) formed strong gels in a number of organic solvents in the presence of TNF. Compounds 14, 16, and 17 formed strong organogels in a variety of organic solvents (table 1). The PBA ester of a cholamide (14) formed a strong CT gel in aromatic hydrocarbon solvents like benzene similar to reported cholamides (compound 5, not a CT gel). ${ }^{31}$ These organogels were characterized by various physical techniques and are discussed in detail in the proceeding sections. Other cholamides like iso-propyl cholamide (compound 8), ${ }^{32}$ a known gelator, do not show gelation if used with the corresponding PBA amide (9) in the tested solvents. However, the PBA esters of cholates $\mathbf{1 6}$ and $\mathbf{1 7}$ do gel higher alcohols and cyclohexanol (table 1). Thus the gelated solvent systems can be changed by modifying the bile acid side chain.

The length of the connection moiety between the pyrene and bile acid part of the gelators plays an important role (viz., compounds 14 and 15). If pyrene-1dodecanoic acid (15) is used instead of PBA, the gelation properties are lost (within the tested solvent range; listed in table 1), $\mathbf{1 4}$ does gel benzene, whereas $\mathbf{1 5}$ does not. This is in line with our previous observations that the presence of pyrene moiety on the bile acid side chain normally leads to loss of gelation properties. ${ }^{24}$

At this point, the role of the bile acid backbone on the gelation is very important. This is further highlighted by observation that a very small variation of the bile acid skeleton will influence gelation. Compound $\mathbf{1 3}$ with one 
Table 1. Gelation profile of compounds 14,16 , and 17 in different organic solvents $\left(\mathrm{C}_{\text {gelator }} 1 \% \mathrm{w} / \mathrm{v}, 1: 1 \mathrm{TNF}\right)$.

\begin{tabular}{lcccc}
\hline Solvent & $\mathbf{1 4}+\mathbf{2 4}^{[\mathrm{a}]}$ & $\mathbf{1 6}+\mathbf{2 4}^{[\mathrm{a}]}$ & $\mathbf{1 7}+\mathbf{2 4}^{[\mathrm{a}]}$ & $\mathbf{2 3}+\mathbf{2 4}^{[\mathrm{a}]}$ \\
\hline Benzene & $\mathrm{G}$ & $\mathrm{S}$ & $\mathrm{S}$ & $\mathrm{S}$ \\
Toluene & $\mathrm{S}$ & $\mathrm{S}$ & $\mathrm{S}$ & $\mathrm{S}$ \\
Dichlorobenzene & $\mathrm{S}$ & $\mathrm{S}$ & $\mathrm{S}$ & $\mathrm{S}$ \\
1-Chlrobenzene & $\mathrm{S}$ & $\mathrm{S}$ & $\mathrm{S}$ & $\mathrm{S}$ \\
Cyclohexane & $\mathrm{S}$ & $\mathrm{GP}$ & $\mathrm{Ss}$ & $\mathrm{S}$ \\
Acetonitrile & $\mathrm{Ss}$ & $\mathrm{S}$ & $\mathrm{S}$ & $\mathrm{P}$ \\
CCl $_{4}$ & $\mathrm{~S}$ & $\mathrm{~S}$ & $\mathrm{~S}$ & $\mathrm{~S}$ \\
Cyclohexanol & $\mathrm{S}$ & $\mathrm{GP}$ & $\mathrm{G}$ & $\mathrm{S}$ \\
1-Decanol & $\mathrm{S}$ & $\mathrm{G}$ & $\mathrm{G}$ & $\mathrm{S}$ \\
Isopropanol & $\mathrm{Ss}$ & $\mathrm{GP}$ & $\mathrm{GP}$ & $\mathrm{Ss}$ \\
Ethanol & $\mathrm{Ss}$ & $\mathrm{Ss}$ & $\mathrm{Ss}$ & $\mathrm{Ss}$ \\
Water & $\mathrm{I}$ & $\mathrm{I}$ & $\mathrm{I}$ & $\mathrm{I}$ \\
\hline
\end{tabular}

[a] = I: Insoluble, S: Solution, Ss: Sparingly Soluble, G: Strong Gel, GP: Gelatinous Precipitate, P: Precipitate

$\mathrm{OH}$ group more than 14, does not form gel with benzene as $\mathbf{1 4}$ does. We believe that the bile acid backbone provides a rigid central core aggregation behaviour of which can be modulated by the substituents on it and linked to it. It seems that the PBA moiety attached at the position 3 of the bile backbone facilitates the multidimensional growth (when TNF is added) of the preliminary aggregates formed, thus leading to gel formation. The pyrene-1-dodecanoic acid esters of bile analogues (compounds 13 and 15), however, failed to gel organic solvents probably due to the lack of right hydrophobichydrophilic balance. These molecules may also be too flexible to have the needed growth of the aggregates when compared to PBA esters.

The pyrene derivatives reported here are functionalized at the alkyl end of the pyrene that is different from pyrene derivatives studied in our earlier reports. ${ }^{25 a-b}$ Our initial discovery of aromatic donor-substituted bile acid derivatives, such as $\mathbf{2 5}$ (chart 2), gelled certain organic solvents in the presence of TNF as the acceptor prompted us to examine the role of the bile acid moiety in these gelators. Some of these pyrene analogues were found to gel even when the bile portion of the molecule was replaced by a simple alkyl chain (e.g., compound 26).

To address this question, a pyrene analogue was synthesized following standard protocols in which the bile acid moiety was replaced by simple alkyl chains connected to the pyrene through an ester linkage (compound 23). This compound formed a deep red CT complex, which remained in solution even after 4 days, no gel formation was observed (table 1). The above gelation findings clearly indicate that pyrene-based gelators need the presence of a bile unit with an ester or amide linkages between them in order to be an efficient gelator.

\subsection{Absorption characteristics}

During the gelation of the compounds 14, 16 and 17, a clear colour change was observed with TNF, caused by an increase in the charge-transfer interaction (CT) during the gelation. This prompted us to study the gelation of these compounds by UV-Visible spectroscopy. Absorption spectroscopic studies were performed with compound 23 in the presence and absence of TNF (conc. of $\mathbf{2 3}$ and TNF is $10 \mu \mathrm{M}$ ). At this concentration no CT band could be seen. However, the band was seen at $0.5 \mathrm{mM}$ of $\mathbf{2 3}$ and TNF.

Similar studies performed for compound $\mathbf{1 7}$ $(0.6 \mathrm{mM})$ with TNF $(0.6 \mathrm{mM})$ showed a band width $\lambda_{\max } 575 \mathrm{~nm}$ (figure 1), which is indicative of the formation of a charge transfer complex. Transformation from sol to gel or from gel to sol has a clear effect on the intensity of the charge transfer band. The charge transfer band intensity decreased when the gel was converted into sol and vice versa. However, there is no

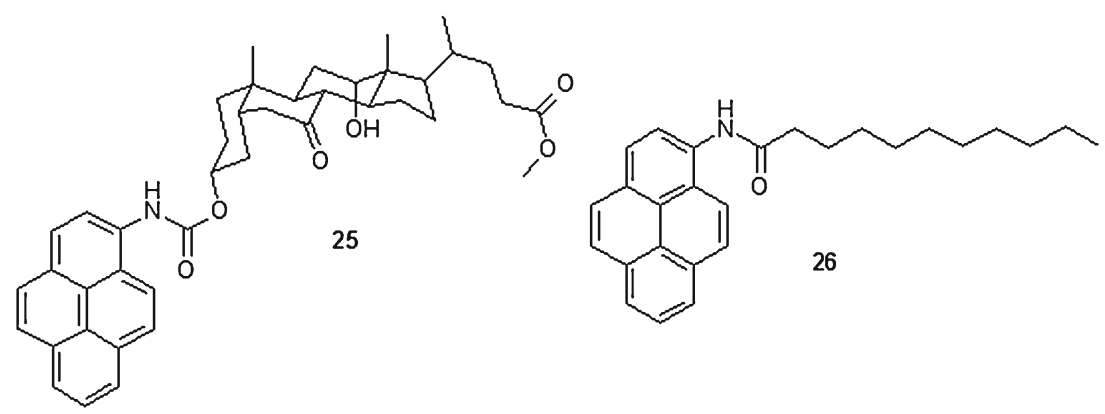

Chart 2. Reference molecules used in this study. 


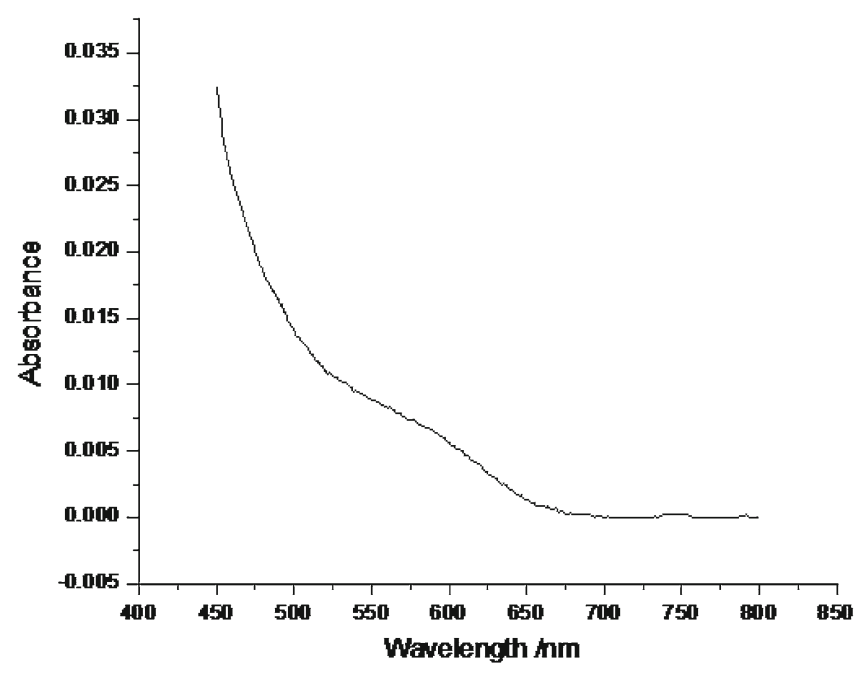

Figure 1. Absorption spectrum of compound $17(0.6 \mathrm{mM})$ with TNF $(0.6 \mathrm{mM})$.

additional band nor change in the $\lambda_{\max }$ was detected between the cycles of sol-to-gel or gel-to-sol.

\subsection{Gel melting}

Although the gels (table 1) were formed by the entropic factors a small contribution from the enthalpy is always present. To dissolve the gelator in a given solvent, heat must be supplied to the system. Once the gel is formed, there exists a temperature at which the gel melts back to form a sol. The gel-sol transition was recorded by

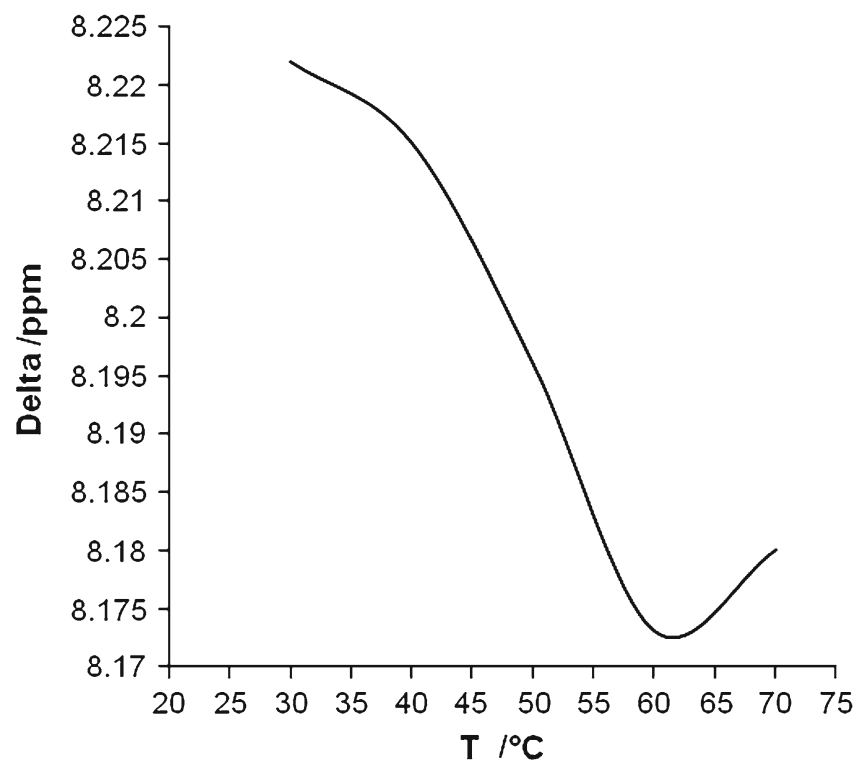

Figure 2. A plot of chemical shift of a pyrene proton $v s$. experimental temperature for $\mathrm{CT}$ gel of compound $\mathbf{1 4}$ $(5.5 \mathrm{mM})$ with TNF $(5.5 \mathrm{mM})$ in benzene $\left[\mathrm{D}_{6}\right]$. the upside down test tube method. The temperature at which the gel started flowing was taken as the transition point. The CT gel $\mathbf{1 4}(1 \% \mathrm{w} / \mathrm{v}$ in benzene, $1: 1$ TNF) melted at $55^{\circ} \mathrm{C}$ and became clear solution at $70^{\circ} \mathrm{C}$. The gels of compound $\mathbf{1 6}$ and $\mathbf{1 7}$ in 1-decanol exhibited slightly lower $\mathrm{T}_{\text {gel }}$ values $\left(42^{\circ} \mathrm{C}\right.$ and $45^{\circ} \mathrm{C}$ respectively) compared to benzene gels of $\mathbf{1 4}$. The $\mathrm{T}_{\text {gel }}$ value observed in this class of organogels is smaller compared to other TNF gels and close to the individual powder melting point of the gelators. The CT gels of 14,16 and $\mathbf{1 7}$ are thermoreversible.

\section{$3.4 \quad{ }^{1} H$ NMR studies}

The sol-gel transition process monitored by NMR showed a significant difference on the proton signals. ${ }^{1} \mathrm{H}$ NMR spectra were recorded for a benzene $\left[\mathrm{D}_{6}\right]$ gel of $\mathbf{1 6}$ at various temperatures. A well-ordered pattern turned into a complex broad pattern when the sol was allowed to gel during the NMR experiment. The transition from gel-sol was studied by plotting the chemical shift (figure 2) and the width of the NMR signals against temperature.

A variable temperature ${ }^{1} \mathrm{H}$ NMR (figure 3 ) is in accordance with the melting studies. At $60^{\circ} \mathrm{C}$, the broad signals observed at low temperatures transformed into sharp patterns. The broadness of the signals at low temperatures is probably attributed to the poor relaxation in the gel state that transformed into a sharp signal when gel-sol transition took place. There was appreciable shift in the $\delta_{\mathrm{NH}}$ of gelator $\mathbf{1 4}$ on going from the sol to gel

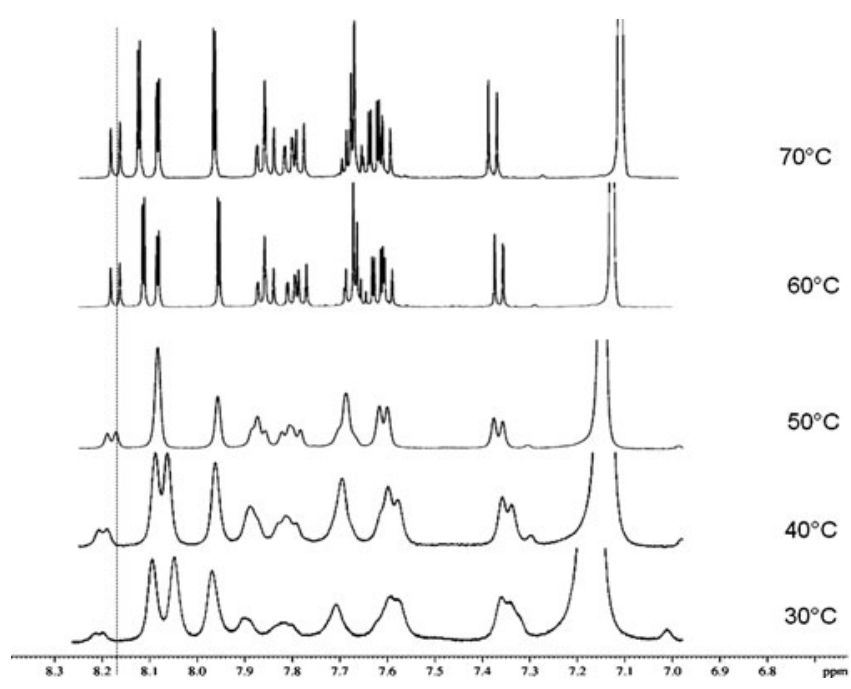

Figure 3. A plot of variable temperature ${ }^{1} \mathrm{H}$ NMR of compound $14(5.5 \mathrm{mM})+\mathrm{TNF}(5.5 \mathrm{mM})$ in benzene $\left[\mathrm{D}_{6}\right]$. Mainly the aromatic portion of the spectrum is shown here. 

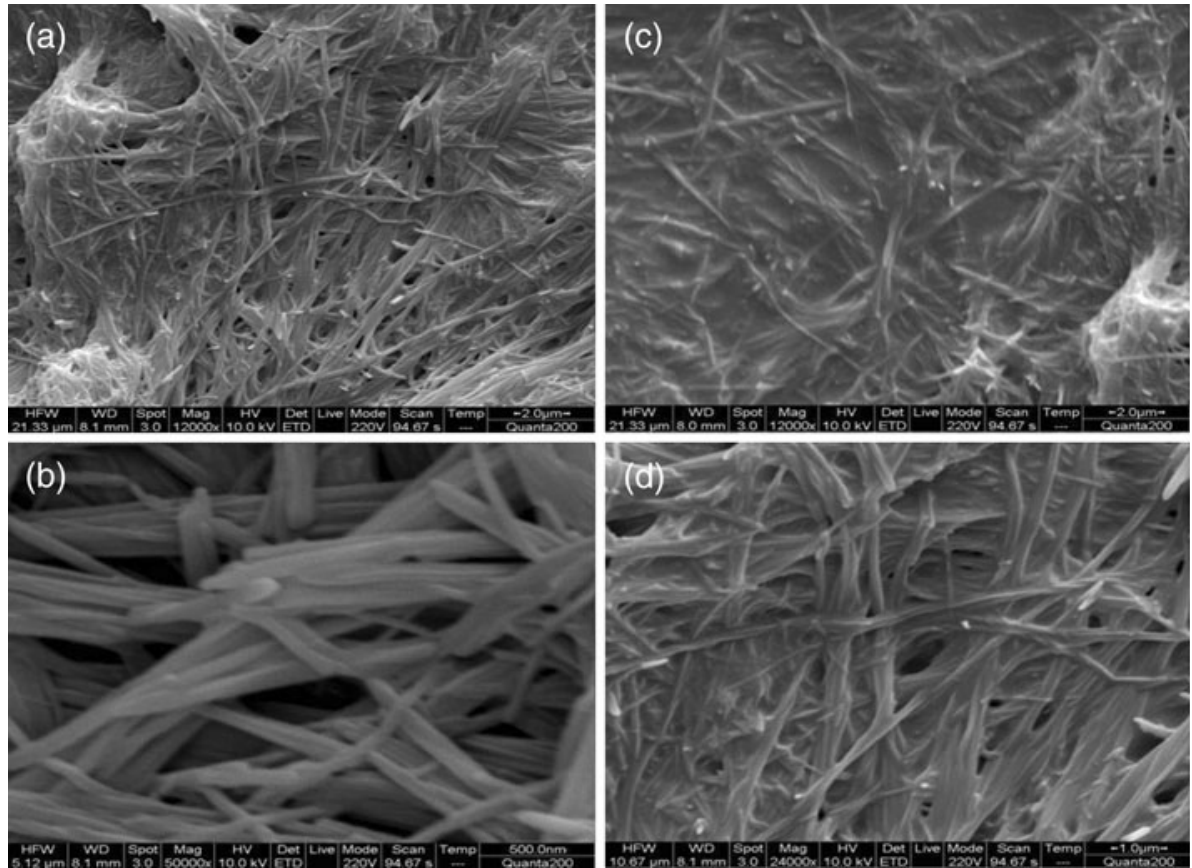

Figure 4. The SEM images of xerogels. a and b: Compound $14+\mathrm{TNF}(1: 1,1 \%$ $\mathrm{w} / \mathrm{v})$ in benzene; $\mathbf{c}$ and $\mathbf{d}$ : Compound $\mathbf{1 7}+\mathrm{TNF}(1: 1 ; 1 \% \mathrm{w} / \mathrm{v})$ in 1-decanol.

phase. This indicated that the side-chain functionality present on the bile acid has a crucial role on the gelation of this class of gelators. In addition, the chemical shifts of methylene groups of butyric acid also shifted considerably during the gelation (compound 14). It appears that the growth of the aggregates has a strong connection with the spacer used between pyrene and bile acid. The TNF is probably acting as an initiator that influences the growth of the aggregates, which in turn leads to gelation of the system. The TNF protons in the gel showed a strong variation in the ${ }^{1} \mathrm{H}$ NMR chemical shifts. It was clearly observed that the doublets of TNF at $8.05(\mathrm{~d}, \mathrm{~J}=2.5 \mathrm{~Hz}, 1 \mathrm{H})$, and $8.11(\mathrm{~d}, \mathrm{~J}=2 \mathrm{~Hz}$, $1 \mathrm{H})$ appears as a broad singlets when they form a gel with compound 14. When the temperature is increased both of the signals merge into a broad singlet at $50^{\circ} \mathrm{C}$ and finally they appear as two doublets after complete melting (figure 3). This implies a strong change in the chemical environment during gel-sol transition.

\subsection{Gel morphology}

The topography of the gels was characterized by the electron microscopy. The SEM images of the xerogels of compounds $\mathbf{1 4}$ and $\mathbf{1 6}$ are shown in the figure 4. The images revealed the presence of infinitely long interconnected rod-like structures with a rod diameter of about
$50 \mathrm{~nm}$. The rods are mostly bundled and the individual rods are rarely seen. These rods reveal no branching and the overall structure can thus be considered as an open-type network. The fractures of the network seen in some parts of the image are probably due to the faster evaporation of the solvent benzene at room temperature during sample preparation.

\subsection{Chirality of the supramolecular aggregates}

The exact relation between molecular chirality and supramolecular chirality is not very clear. In some cases, the racemic and achiral molecules form chiral aggregates. ${ }^{33}$ Many of the reported hydrogels derived from deoxycholate and its conjugates have been shown to exhibit supramolecular helicity. ${ }^{34}$ The gelators $\mathbf{1 4}$, 16, and $\mathbf{1 7}$ have been derived from homochiral bile acid systems. However, no such supramolecular helicity or chiral signatures could be extracted from SEM analysis.

\section{Conclusions}

We have synthesized different pyrene and bile acidpyrene derivatives with interest of their self-association properties in various organic and aqueous solvents. Among the compounds prepared the PBA esters of bile 
acid analogues were found to gel many organic solvents in the presence of TNF through the formation of a charge transfer complex. The gels obtained are optically transparent and deep red in colour attributed to the charge transfer band. The addition of TNF seems to grow and strengthen the smaller aggregates ultimately leading to the gelation of the solvents. A structuregelation property studies revealed that the length of the alkyl spacer between pyrene and ester carbonyl situated at position 3 of the bile acids is one of the limiting factors for the gelation. The side chain functionality and the structure is the second factor which can be available for fine tuning the gelation abilities of this class of organogelators. The replacement of a bile acid moiety by a simple alkyl chain highlighted the importance of bile acid backbone in this gelator class. The gels are thermoreversible and stable to several cycles of melting and cooling. A highly entangled rod-like structures were seen from the xerogels with no signature of supramolecular chirality.

\section{Acknowledgements}

Financial support from the Academy of Finland (grants 106998 and 212588) is gratefully acknowledged. Thanks are due to special laboratory technician Reijo Kauppinen for helping NMR measurements and to special laboratory technician Mirja Lahtiperä for mass spectrometry (MS). We also thank the Institute Nano Initiative (INI) at the Indian Institute of Science (IISc), Bangalore, India, for providing SEM facility. The IISc group thanks the Department of Science and Technology, New Delhi, for financial support in the form of a J.C. Bose Fellowship to UM.

\section{References}

1. 2006 Molecular gels: Materials with self-assembled fibrillar networks (eds.) R G Weiss and P Terech (Netherlands: Kluwer Academic Publication Springer)

2. (a) Terech P 1997 Low-molecular weight organogelators in Specialist surfactants (ed.) I D Robb (Glasgow: Blackie Academic and Professional) p. 208; (b) van Esch J, Schoonbeek F, De Loos M, Veen E M, Kellog R M, Feringa B L 1999 Low molecular weight gelators for organic solvents in supramolecular science: where it is and where it is going (eds.) R Ungaro and E Dalcanale (Dordrecht: Kluwer Academic Publishers) p. 233

3. (a) Terech P, Weiss R G 1997 Chem. Rev. 97 3133; (b) Liu X Y, Sawant P D 2002 Adv. Mater. 14 421; (c) Liu X Y, Sawant P D 2001 Appl. Phys. Lett. 793518

4. Abdallah D J, Weiss R G 2000 Adv. Mater. 121237

5. (a) Lo N P, Roland R, Emiliano F, Marco R, Francesca L, Emiliano C, Moira A, Barry N, Piero W B 2007 J.
Phys. Chem. B 111 11714; (b) Valkonen A, Lahtinen M, Virtanen E, Kaikkonen S, Kolehmainen, E 2004 Biosens. Bioelectron. 201233

6. (a) Yoshikawa I, Yanagi S, Yamaji Y, Araki K 2007 Tetrahedron 63 7474; (b) Park S M, Kim B H 2008 Soft Mater. 4 1995; (c) Fried M G, Bloomfield V A 1984 Biopolymers 23 2141; (d) Starr F W, Sciortino F 2006 J. Phys.: Condens. Matter. 18 L347

7. Atsbeha T, Bussotti L, Cicchi S, Foggi P, Ghini G, Lascialfari L, Marcelli A 2011 J. Mol. Struct. 993(1-3) 459-463

8. Murdan S 2005 Expert opinion on drug delivery 2489

9. Placin F, Desvergne J-P, Cansell F 2000 J. Mater. Chem. 102147

10. (a) de Vries E J, Kellogg R M 1993 J. Chem. Soc., Chem. Commun. 238; (b) Sobna J-E S, Fages F 1997 Chem. Commun. 327; (c) Hanabusa K, Maesaka Y, Kimura M, Shirai H, 1999 Tetrahedron Lett. 40 2385; (d) Kimura T, Yamashita T, Koumoto K, Shinkai S 1999 Tetrahedron Lett. 40 6631; (e) Bhattacharya S, Acharya S N G 1999 Chem. Mater. 113121

11. Abdallah D J, Weiss R G 2000 Langmuir 16352

12. (a) van Esch J, Feringa B L 2000 Angew. Chem. 112 2351; (b) Ren X, Yu W, Zhang Z, Xia N, Fu G, Lu X, Wang W 2011 Colloids Surf., A 375 156; (c) Yagai S, Aonuma H, Kikkawa Y, Kubota S, Karatsu T, Kitamura A, Mahesh S, Ajayaghosh A 2010 Chem. Eur. J. 16 8652; (d) Samanta S K, Pal A, Bhattacharya S 2009 Langmuir 25 8567; (e) Simalou O, Xue P, Lu R 2010 Tetrahedron Lett. 51, 3685; (f) Yang X, Zhang G, Zhang D, Zhu D 2010 Langmuir 26 11720; (g) Jang K, Ranasinghe A D, Heske C, Lee D-C 2010 Langmuir 26 13630; (h) Babu S S, Kartha K K, Ajayaghosh A 2010 J. Phys. Chem. Lett. 13413

13. (a) Kato T 2002 Science 295 2414; (b) Wan J-H, Mao L-Y, Li Y-B, Li Z-F, Qiu H-Y, Wang C, Lai G-Q 2010 Soft Mater. 63195

14. Tung S-H, Huang Y-E, Raghavan S R 2008 Soft Mater. 41086

15. Encyclopedia of Supramolecular Chemistry (eds.) J L Atwood and J W Steed (New York: Marcel Dekker)

16. Naota T, Koori H 2005 J. Am. Chem. Soc. 1279324

17. Wu J, Yi T, Shu T, Yu M, Zhou Z, Xu M, Zhou Y, Zhang H, Han J, Li F, Huang C 2007 Angew. Chem., Int. Ed. 47 1063

18. (a) Murata K, Aoki M, Suzuki T, Harada T, Kawabata H, Komori T, Ohseto F, Ueda K, Shinkai S 1994 J. Am. Chem. Soc. 116 6664; (b) Ayabe M, Kishida T, Fujita N, Sada K, Shinkai S 2003 Org. Biomol. Chem. 1 2744; (c) Ahmed S A, Sallenave X, Fages F, Mieden-G G, Muller W M, Muller U, Vogtle F, Pozzo J-L 2002 Langmuir 18 7096; (d) Frkanec L, Jokic M, Makarevic J, Wolsperger K, Zÿinic M 2002 J. Am. Chem. Soc. 1249716

19. Wang C, Zhang D, Xiang J, Zhu D 2007 Langmuir 23 9195

20. Haino T, Saito H 2010 Aust. J. Chem. 63640

21. (a) Saji T, Hoshino K, Ishii Y, Goto M 1991 J. Am. Chem. Soc. 113 450; (b) Tsuchiya K, Orihara Y, Kondo Y, Yoshino N, Ohkubo T, Sakai H, Abe M 2004 J. Am. Chem. Soc. 126 12282; (c) Medina J C, Gay I., Chen Z, Echegoyen L, Gokel G W 1991 J. Am. Chem. Soc. 
113 365; (d) Nayak M K, Kim B-H, Kwon J E, Park S, Seo J, Chung J W, Park S Y 2010 Chem. Eur. J. 16 7437

22. Fuhrhop J H, Koening J 1994 Membranes and molecular assemblies: The synkinetic approach (Cambridge: Royal Society of Chemistry)

23. (a) Hanabusa K, Yamada M, Kimura M, Shirai H 1996 Angew. Chem., Int. Ed. 35 1949; (b) Hanabusa K, Shimura K, Hirose K, Kimura M, Shirai H 1996 Chem. Lett. 885

24. (a) van Esch J, Kellogg R M, Feringa B L 1997 Tetrahedron Lett. 38 281; (b) van Esch J, De Feyter S, De Schryver F, Kellogg R M, Feringa B L 1997 Chem. Eur. J. 31238

25. (a) Maitra U, Kumar P V, Chandra N, d'Souza L J, Prasanna M D, Raju A R 1999 Chem. Commun. 595; (b) Babu P, Sangeetha N M, Kumar P V, Maitra U, Rissanen K, Raju A R 2003 Chem. Eur. J. 9 1922; (c) Mukhopadhyay S, Maitra U 2004 Curr. Sci. 87 1666
26. (a) Chang Y L, West M-A, Fowler F W, Lauher J W 1993 J. Am. Chem. Soc. 115 5991; (b) Das A, Ghosh S 2010 Chem. Eur. J. 16 13622; (c) Allix F, Curcio P, Pham Q N, Pickaert G, Jamart-Grégoire B 2010 Langmuir 2616818

27. Hahma A, Bhat S, Leivo K, Linnanto J, Lahtinen M, Rissanen K 2008 New J. Chem. 321438

28. Cortese F, Bauman L 1935 J. Am. Chem. Soc. 571393

29. (a) Bhat S, Maitra S 2007 Tetrahedron 63 7309; (b) Sangeetha N M, Balasubramanian R, Maitra U, Ghosh S, Raju A R 2002 Langmuir 187154

30. Dias J R, Gao H, Kolehmainen E 2000 Spectrochim. Acta, A $\mathbf{5 6} 53$

31. Willemen $\mathrm{H} \mathrm{M}$, Vermonden $\mathrm{T}$, Marcelis A $\mathrm{T} \mathrm{M}$, Sudhölter E J R 2002 Langmuir 187102

32. Hishikawa Y, Sada K, Watanabe R, Miyata M, Hanabusa K 1998 Chem. Lett. 795

33. Phakomov S, Hammer R P, Mishra B K, Thomas B N 2003 Proc. Natl. Acad. Sci. U.S.A. 1003040

34. (a) Rich A, Blow D M 1958 Nature 182 423; (b) Rich A, Blow D M 1960 J. Am. Chem. Soc. 823566 